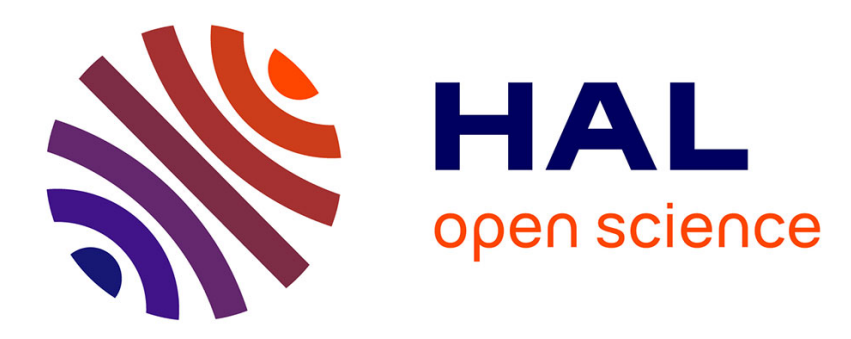

\title{
Inhomogeneous Financial Networks and Contagious Links
}

Hamed Amini, Andreea Minca

\section{To cite this version:}

Hamed Amini, Andreea Minca. Inhomogeneous Financial Networks and Contagious Links . 2014. hal-01081559

\section{HAL Id: hal-01081559 \\ https://hal.inria.fr/hal-01081559}

Preprint submitted on 9 Nov 2014

HAL is a multi-disciplinary open access archive for the deposit and dissemination of scientific research documents, whether they are published or not. The documents may come from teaching and research institutions in France or abroad, or from public or private research centers.
L'archive ouverte pluridisciplinaire HAL, est destinée au dépôt et à la diffusion de documents scientifiques de niveau recherche, publiés ou non, émanant des établissements d'enseignement et de recherche français ou étrangers, des laboratoires publics ou privés. 


\title{
Inhomogeneous Financial Networks and Contagious Links*
}

\author{
Hamed Amini ${ }^{\dagger}$ and Andreea Minca ${ }^{\ddagger}$
}

October 31, 2014

\begin{abstract}
We propose a framework for testing the possibility of large cascades in financial networks. This framework accommodates a variety of specifications for the probabilities of emergence of 'contagious links', where a contagious link leads to the default of a bank following the default of its counterparty. These are the first order contagion probabilities and depend on the shock propagation mechanism under consideration. When the cascade represents an insolvency cascade, and under complete observation of balance sheets, the first order contagion probabilities follow from the distribution of recovery rates.

Under general contagion mechanisms and incomplete information, the financial network is modeled as an inhomogenous random graph in which only some of the banks' characteristics are observable. We give bounds on the size of the first order contagion and testable conditions for it to be small. For power-law financial networks, we also give a condition so that the higher order cascade dies out.
\end{abstract}

Keywords: systemic risk, default contagion, financial stability, interbank network, phase transitions, sharp threshold, complex networks, power-law random graph.

\section{Introduction}

The literature on network models for systemic risk has seen recently a sharp increase across different disciplines, see e.g., $[8,45,2,33,36,18,19]$. Network models for distress propagation among institutions/sectors/regions have mainly sought to understand the relation between network structure and systemic risk. The resulting insights are mostly qualitative in nature. In a recent speech on this topic, Janet Yellen notes "With a few narrow exceptions, they treat all market participants as similar in size and in range of activities, and they use relatively simplistic network structures. " [50].

Empirical studies (see Figure 1) point to the diversity of network structures of real-world banking systems: from scale-free structures as in [22, 28] to centralized networks [43] or

\footnotetext{
*Part of this work was accomplished while the authors were visiting Isaac Newton Institute for Mathematical Sciences in Cambridge for the program on "Systemic Risk: Mathematical Modelling and Interdisciplinary Approaches". We thank them for their hospitality. We also thank Rama Cont and Nikolaos Fountoulakis for helpful discussions.

${ }^{\dagger}$ Swiss Finance Institute @ EPFL, Quartier UNIL-Dorigny, Extranef 249, 1015 Lausanne, Switzerland, email: hamed.amini@epfl.ch

${ }^{\ddagger}$ School of Operations Research and Information Engineering, Cornell University, Ithaca, NY 14850, USA, email: acm299@cornell.edu
} 
core-periphery structures in [30]. Some of these networks can well be approximated with few classes of banks, core and peripheral and are homogenous within each class. Others, as the UK banking system shown in Figure 1, are highly heterogenous in both the connectivity as well as in the the size of the banks' balance sheets. In [22, 28], the connectivity is shown to follow a power law distribution across banks. As it is well known in the epidemics and percolation literature, e.g., [4, 5, 6, 20,34, 40, 39, 41, 44, 46, 49], the network structure plays an essential role in the propagation of distress.
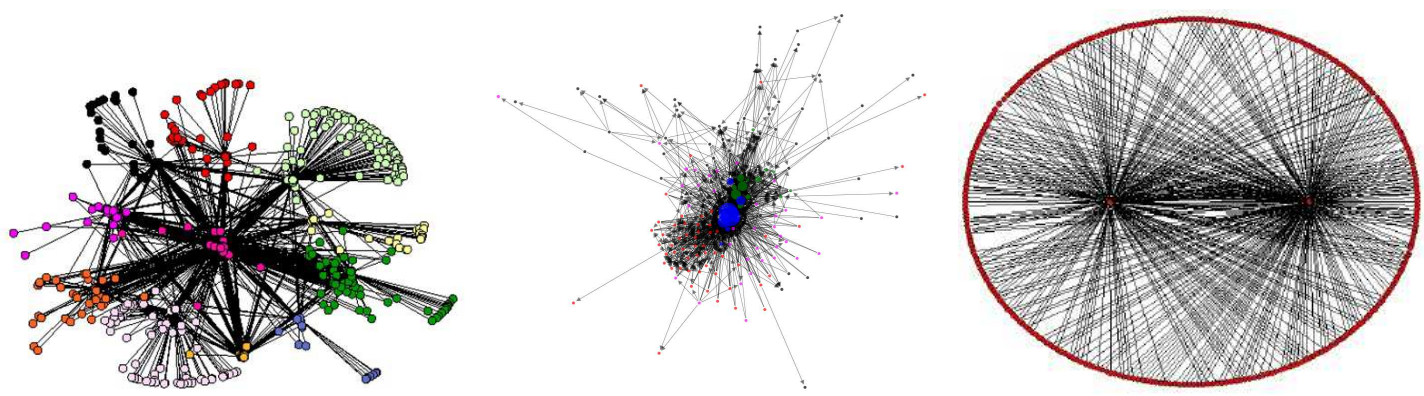

Figure 1: Structure of interbank networks. From left to right: Austria, scale-free structure [22]; UK, sparse inhomogenous structure [16]; Switzerland, sparse and centralized structure [43].

Another critical point to consider in a model of financial contagion is the available information. In some few cases the modeler has complete information on interbank linkages. As pointed out in [13], there is in general only partial information on the interbank network and only aggregate data, for example on the total size of the assets and liabilities, is available. From this point of view, a random network, i.e., a set of networks consistent with the observable aggregates and a probability distribution over these networks, captures well the partial information structure.

Random networks in which the characteristics of the banks can be prescribed provide the flexibility needed to model financial networks. These characteristics may have different degrees of heterogeneity and may be calibrated to real-world data. Moreover, such models are amenable to analysis of the size of contagion following an initial shock and yield testable conditions for the possibility of large default cascades. Such probabilistic models can thus provide powerful tools for financial regulation.

In previous work [8], we considered a model of default propagation in a financial network modeled as a random network with prescribed connectivity, exposure and capital sequences. The main result in [8] is the asymptotic size of contagion in terms of these sequences. Moreover, in [8], we introduce the concept of contagious exposures, i.e., those exposures that lead to the default of a bank in case the counterparty defaults and show that the topology of the subgraph of contagious links determines whether the cascade is large scale or not. It turns out that, for large networks, the condition for large cascades can be characterized in terms of observable quantities such as the number of contagious exposures and the connectivity. This condition can be used in a stress testing framework [7]. Under different shocks applied to the entire sequence of capital and exposures, the topology of the subgraph of contagious links changes and a phase transition occurs for sufficiently large shocks. Namely, the system passes in a regime when large scale cascades are possible.

Retaining the focus on testable conditions for large cascades, the current paper proposes multiple extensions of [8]: 
- We allow for random recovery rates for the exposures to defaulted banks, which can be thought of as the uncertain recovery rates by the end of the cascade. While it is common in the literature to derive recovery rates from an elegant fixed point equation [35], this relies on the assumption that all debts are instantaneously cleared, unlikely to hold in reality. At the time of the initial shock, recovery rates are uncertain. ${ }^{1}$ In this paper we model recovery rates as random variables (who satisfy some cash-flow consistency conditions). This captures the uncertainty about the value of the defaulted bank, the amount of deposits with higher seniority and also the intertemporal aspect of repaying to the creditors.

- The probability distribution for the recovery rates induces a (first-order) contagion probability matrix, which gives for each pair of nodes the probability that there is a contagious link between them. In a stress testing framework for insolvency contagion, one can rely on the specification of the distribution of the recovery rates, of the asset shocks and on data on interbank exposures to compute this contagion probability matrix.

However, the concept of contagious links is by no means restricted to insolvency contagion and a different model of contagion induces a different probability matrix. From this point of view, our paper can be thought of as a framework for testing the large scale contagion under different propagation mechanisms: there can by a contagious link between two nodes if there is a critical exposure as in [8], a critical OTC cash-flow as in [27] or if the funding structure leads to a contagion of bank runs [14], etc. Moreover, the contagious links induced by multiple contagion mechanisms may co-exist [12, 37, 38].

As pointed out in [37], contagion through the network of exposures becomes significant in presence of heterogeneity and rollover risks. The presence of rollover risks can be captured in our model by introducing contagious links among banks that rely on unstable short term funding.

- Given a general contagion probability matrix, we give sufficient conditions such that the size of first order cascade, due to contagious links and which is the primary source of the cascade, to be small. Some of these bounds are known for the homogeneous undirected networks in the context of SIR epidemics and bond percolation, see [24, $31,32,34]$. These are unrealistic settings in financial networks. Our first technical contribution is to extend these results for directed and heterogenous networks. We also give several examples on how these bounds can be used for different topologies of the financial network.

- We consider an incomplete information setting where we only observe characteristics or "types" of market participants in the financial system. The inhomogeneity of the graph is assumed within these types. A particularly relevant set of types is that of core/peripheral bank, see Example 13. In this case, we give a simple and testable condition for the financial network to be resilient, i.e., that starting from a small shock, the final size of the cascade is small. However, the model allows for rather general sets of types of the financial institutions, both finite as well as (countably or uncountably) infinite. An example of an uncountably infinite type space could be types related to the total liability and the total asset of each individual bank in the financial network; as in Example 14. Extending the previous results on percolation in undirected random graphs in $[10,11,40]$ to inhomogeneous directed random graphs, we give a necessary

\footnotetext{
${ }^{1}$ Recovery rates after the failure of Lehman were around eight cents on the dollar [42]. The recovery rates after three months were around ten cents on the dollar.
} 
and sufficient condition such that the size of the first order cascade to be small. This condition marks a phase transition in the size of the first order cascade.

- We show that for financial networks with infinite second order moment for the degree distribution a fraction of initial defaults that tends to zero may lead to a positive fraction of final defaults, so that the default amplification may be unbounded.

- Finally, we consider a power-law degree distribution for the financial network, which may have infinite second order moment, and give a precise condition under which we do not have a higher order cascade and the final size of the cascade is the same as the size of the first order cascade. Under this condition, it suffices to monitor the size of the first order cascade to ensure the stability of the financial network. We characterize the subcritical regime of the cascade in terms of the distribution of the degrees in the network. The asymmetry in connectivity induced by a power law distribution plays a critical role in financial contagion and may lead to effects at the level of the whole system, see e.g., [1].

The paper is organized as follows. In Section 2 we introduce a model of financial contagion on a given network, with random recovery rates. In Section 3, we give bounds on the size of the first order cascade in terms of contagion probability matrix. In Section 4 we introduce the random graph model and bound the size of the (random) subgraph of contagious links. In Section 5 we give conditions on the connectivity for the size of the cascade to be equal to the size of the first order cascade. Section 6 concludes. The proofs of all lemmas and theorems are provided in Appendix A.

Basic notations. We let $\mathbb{N}$ be the set of non-negative integers. For non-negative sequences $x_{n}$ and $y_{n}$, we write $x_{n}=O\left(y_{n}\right)$ if there exist $N \in \mathbb{N}$ and $C>0$ such that $x_{n} \leq C y_{n}$ for all $n \geq N$, and $x_{n}=o\left(y_{n}\right)$ (or $\left.x_{n} \ll y_{n}\right)$, if $x_{n} / y_{n} \rightarrow 0$, as $n \rightarrow \infty$. Let $\left\{X_{n}\right\}_{n \in \mathbb{N}}$ be a sequence of real-valued random variables on a probability space $(\Omega, \mathbb{P})$. If $c \in \mathbb{R}$ is a constant, we write $X_{n} \stackrel{p}{\longrightarrow} c$ to denote that $X_{n}$ converges in probability to $c$. That is, for any $\epsilon>0$, we have $\mathbb{P}\left(\left|X_{n}-c\right|>\epsilon\right) \rightarrow 0$ as $n \rightarrow \infty$. Let $\left\{a_{n}\right\}_{n \in \mathbb{N}}$ be a sequence of real numbers that tends to infinity as $n \rightarrow \infty$. We write $X_{n}=o_{p}\left(a_{n}\right)$, if $\left|X_{n}\right| / a_{n}$ converges to 0 in probability. Additionally, we write $X_{n}=O_{p}\left(a_{n}\right)$, to denote that for any positive sequence $\omega(n) \rightarrow \infty$, we have $\mathbb{P}\left(\left|X_{n}\right| / a_{n} \geq \omega(n)\right)=o(1)$. If $\mathcal{E}_{n}$ is a measurable subset of $\Omega$, for any $n \in \mathbb{N}$, we say that the sequence $\left\{\mathcal{E}_{n}\right\}_{n \in \mathbb{N}}$ occurs with high probability (w.h.p.) if $\mathbb{P}\left(\mathcal{E}_{n}\right)=1-o(1)$, as $n \rightarrow \infty$. Also, we denote by $\operatorname{Be}(p)$ a Bernoulli distributed random variable whose probability of being equal to 1 is $p$. $\operatorname{Bin}(k, p)$ denotes a binomial distribution corresponding to the number of successes of a sequence of $k$ independent Bernoulli trials each having probability of success $p$. We will suppress the dependence of parameters on the size of the network $n$, if it is clear from the context.

\section{Network models of banking systems}

At a fixed time, a financial system is represented as a set $[n]:=\{1, \ldots, n\}$ of financial institutions (banks) that intermediate credit among end-users. Banks hold claims on each other. We model these claims by a liability matrix $L_{i j}$. For any two financial institutions $i$ and $j, L_{i j}$ represents the total liability of $i$ to $j$. This represents the maximum loss related to direct claims, incurred by $j$ upon the failure of $i$. The total value of claims held by end-users on bank $i$ (deposits) is given by $D_{i}$. 


\begin{tabular}{|c|c|}
\hline External assets & Deposits \\
$X_{i}$ & $D_{i}$ \\
\cline { 1 - 1 }$\zeta_{i} X_{i}$ - loss on assets & Interbank liabilities \\
\cline { 1 - 2 } & $L_{i}=\sum_{j \in[n]} L_{i j}$ \\
Interbank assets & \\
\cline { 2 - 2 }$A_{i}=\sum_{j \in[n]} L_{j i}$ & Capital \\
& $C_{i}$ \\
\hline Assets & $\zeta_{i} X_{i}$ - loss on capital \\
\hline
\end{tabular}

Table 1: Stylized balance sheet of bank $i$ after shock.

The total value of interbank assets of bank $i$ is given by

$$
A_{i}:=\sum_{j \in[n]} L_{j i},
$$

while the total value of interbank liabilities of $i$ is given by

$$
L_{i}:=\sum_{j \in[n]} L_{i j} .
$$

The total value of claims held by bank $i$ on end-users (external assets) is denoted by $X_{i}$. We now consider that the external assets of bank $i$ receive a shock $\zeta_{i}$, proportional to the external asset. Let $C_{i}$ be the capital of bank $i$ after the shock, defined as the total value of assets minus the total value of liabilities:

$$
C_{i}:=X_{i}\left(1-\zeta_{i}\right)+A_{i}-L_{i}-D_{i} .
$$

Table 1 shows a stylized balance sheet of bank $i$ after shock.

A bank $i$ is said to be in fundamental default if its capital after the shock is negative

$$
C_{i}<0 .
$$

We let the set of fundamental defaults

$$
\mathcal{D}_{0}=\left\{i \in[n] \mid C_{i}<0\right\} .
$$

Given the sequence of assets and liabilities in the network, the set of fundamental defaults depends only on the shock. We call small shock regime, the regime under which the number of fundamental defaults is small compared to the size of the network.

A bank in default will not be able to repay in full its liabilities. In most of the literature on interbank contagion, see e.g. [35, 9], the recovery rates are considered fully proportional to the asset size of the defaulted bank, but in reality this is too optimistic since liquidation takes time and the valuation of the assets is uncertain. In the following, we denote by $\mathcal{R}=\left(R_{i j}\right)$ the matrix of recovery rates, where $R_{i j}$ denotes the recovery rate of the liability of $i$ to $j$. Since banks are required to hold minimal capital reserves, these recovery rates are bounded from below by a constant $\underline{\mathrm{R}} \in[0,1]$. We assume that the actual recovery rate 
of the liability of $i$ to $j$ (in case of default of $i$ ) is given by $\underline{\mathrm{R}}+\mathcal{E}_{i j}$, where $\mathcal{E}_{i j}$ captures all the uncertainly about the value of the defaulted bank, the amount of deposits with higher seniority and also the intertemporal aspect of repaying to the creditors. Hence we have $R_{i j}=1$ if $i$ is solvent (not in default) and $R_{i j}=\underline{\mathrm{R}}+\mathcal{E}_{i j}$ if $i$ is in default. In particular, in the following we consider that $\left\{\mathcal{E}_{i j}\right\}_{i, j \in[n]}$ are random variables, such that the support of the distribution of $R_{i j}$ satisfies $\operatorname{supp}\left(R_{i j}\right) \subseteq\left[\underline{R}, \bar{R}_{i}\right]$ for some $0 \leq \underline{R} \leq \bar{R}_{i} \leq 1$, which satisfy some cash-flow consistency condition (see the remark below). For tractability, we consider that $\mathcal{E}_{i j}$ are independent (not necessarily identically distributed).

Remark 1. Note that since an institution cannot pay more than its external assets plus what it recovered from its debtors, the recovery rates of each institution $i \in[n]$ must satisfy the following constraints

$$
X_{i}\left(1-\zeta_{i}\right)+\sum_{j \in[n]} L_{j i} R_{j i} \geq \sum_{j \in[n]} L_{i j} R_{i j} .
$$

In order to satisfy the above constraint we can take for example a conservative approach and consider the worst case scenario in which the recovery rates of a defaulted bank are computed under the assumption of minimum recovery rate for its own interbank assets. More precisely, assume that for all $i=1, \ldots, n$

$$
X_{i}\left(1-\zeta_{i}\right)+A_{i} \underline{R} \geq L_{i} \bar{R}_{i} .
$$

In particular, if $\underline{R}=0$ then it suffices to set $\bar{R}_{i}=\frac{X_{i}\left(1-\zeta_{i}\right)}{L_{i}} \wedge 1$ so that $\left[\underline{R}, \bar{R}_{i}\right]$ always exists and one can always choose the maximal such an interval. Note, moreover, that in this case $\bar{R}_{i}$ depends on the bank $i$ only through its characteristics $L_{i}$ and $X_{i}$. The recovery rate $R_{i j}$ follows a distribution which depends only the characteristics of bank $i$.

Following the fundamental defaults, there is a default cascade that reaches the following fixed point equation.

Definition 2 (Cascade fixed point set). Define $\mathcal{D}^{*}$ as the set of institutions whose capital is insufficient to absorb losses due to defaulted institutions

$$
\mathcal{D}^{*}=\mathcal{D}^{*}(\mathcal{R})=\left\{i \in[n] \mid C_{i}<\sum_{j \in \mathcal{D}^{*}}\left(1-R_{j i}\right) L_{j i}\right\} .
$$

The cascade has in general multiple fixed points, as shown by the next lemma. The smallest fixed point set corresponds to the smallest number of defaults and can be reached by assuming a priori that all banks pay in full and then updating the default set: as we learn that some banks default, then some debtor banks will not repay in full. If the recovery rates are too small, i.e., the loss rates are too large, then the creditor banks may default and so on. The largest fixed point corresponds to the largest number of defaults. We assume a priori that all banks default. If there are banks that are able to pay their debts even if all their debtors are in default, then we update the default set by removing these banks, and so on. In practice, there may be no such bank that is able to pay all its debts when its debtors are all in default. In this case, the default set consisting of all banks is the largest fixed point of the cascade.

Lemma 3. There exists largest and smallest cascade fixed point sets $\underline{\mathcal{D}}^{*}, \overline{\mathcal{D}}^{*}$ satisfying (3) such that any cascade fixed point set $\mathcal{D}^{*}$ is included in $\overline{\mathcal{D}}^{*}$ and includes $\underline{\mathcal{D}}^{*}$, i.e., we have

$$
\mathcal{D}_{0} \subseteq \underline{\mathcal{D}}^{*} \subseteq \mathcal{D}^{*} \subseteq \overline{\mathcal{D}}^{*} \subseteq[n]
$$


In the reminder of the paper we only consider the smallest cascade fixed point set $\mathcal{D}^{*}=\underline{\mathcal{D}}^{*}$ which, as the proof of Lemma 3 shows, can be obtained iteratively in at most $n-1$ steps $\left(\underline{\mathcal{D}}^{*}=\mathcal{D}_{n-1}\right)$ by starting from $\mathcal{D}_{0}$ and setting at step $k=1, \ldots, n-1$ :

$$
\mathcal{D}_{k}:=\left\{i \in[n] \mid C_{i}<\sum_{j \in \mathcal{D}_{k-1}}\left(1-R_{j i}\right) L_{j i}\right\} .
$$

As we will show in Section 5, in the small shock regime, the spread of contagion in a large network relies on the existence of a large component connected by contagious links:

Definition 4 (Contagious link). We say that $i \rightarrow j$ is contagious if and only if the default of institution $i$ triggers the default of $j$, i.e., if the loss induced by the default of $i$ on $j$ is larger than the capital of $j$ :

$$
\left(1-R_{i j}\right) L_{i j}>C_{j}
$$

In the following, we fix a probability space $(\Omega, \mathcal{F}, \mathbb{P})$ that models the uncertainly in the financial system. We let $\beta_{i j}$ denote the probability that a link from $i$ to $j$ (with $L_{i j}>0$ ) becomes contagious. Given the liability matrix and the realized shocks (in stress scenarios), the randomness stems from the uncertain recovery rates and we have:

$$
\beta_{i j}:=\mathbb{P}\left(R_{i j}<\frac{L_{i j}-C_{j}}{L_{i j}}\right) .
$$

The subgraph of the financial network constituted of contagious links will be a primary source for the propagation of default cascades: the larger this subgraph, the larger the size of default contagion.

We call first order cascade the contagion carried through the contagious links. More precisely, we denote by $\mathcal{C}_{1}$ the set of banks that can be reached solely by contagious links, starting form the set of fundamental defaults, i.e.,

$$
\mathcal{C}_{1}=\left\{j \in[n] \mid \exists k \geq 1,\left(j_{0} \in \mathcal{D}_{0}, j_{1}, \ldots, j_{k}=j\right), j_{\ell-1} \rightarrow j_{\ell} \text { is contagious } \forall \ell=1, \ldots, k\right\} .
$$

We refer to $\left|\mathcal{C}_{1}\right|$ as the size of the first order cascade. We say that the cascade is small if $\left|\mathcal{C}_{1}\right|=o_{p}(n)$, i.e., the fundamental shock does not lead to a positive fraction of defaults through contagious links. Note that $\mathcal{C}_{1}$ is a random set which depends on $\mathcal{D}_{0}$ and the recovery rates $\mathcal{R}$. Clearly this set contains the set of fundamental defaults and, all banks in this set, by definition of contagion links, are guaranteed to default, so we have

$$
\mathcal{D}_{0} \subseteq \mathcal{C}_{1} \subseteq \mathcal{D}_{n-1}
$$

We call the higher order cascade the cascade that is not carried through contagious links: $\mathcal{D}_{n-1} \backslash \mathcal{C}_{1}$ represent the nodes that default by the higher order cascade. A critical question is regarding the relation between the size of the set $\mathcal{C}_{1}$ and the size of final set of defaults $\mathcal{D}_{n-1}$. In the next two sections, we state some necessary and sufficient conditions on the matrix $B:=\left(\beta_{i j}\right)_{i, j \in[n]}$ for the size of first order cascade in the small shock regime to be small. Namely, we give bounds on the number of institutions in $\mathcal{C}_{1}$. It turns out that bounding the size of $\mathcal{C}_{1}$ is critical to bounding the final number of defaults in the system. We will show in Section 5 (see Theorem 22), that if the size of $\mathcal{C}_{1}$ is smaller than some critical parameter then the final size of cascade will be small. 
We end this section by the following remark. The concept of contagious links in financial networks can be defined more generally than the contractual liabilities for which the loss exceeds the capital of the creditor bank, as in the condition of Definition 4. In general there could be a contagious link between $i$ and $j$ even in absence of liability between $i$ and $j$, for example due to common asset holdings, see e.g., [23, 25, 29] or information contagion [3].

\section{Bounds in general networks}

In this section we state some general conditions on the matrix $B=\left(\beta_{i j}\right)_{i, j \in[n]}$ for the size of the first order cascade, i.e., $\left|\mathcal{C}_{1}\right|$, to be small.

We begin by giving a condition on the maximum row sum of the matrix $B$ which ensures that the amplification $\left|\mathcal{C}_{1}\right| /\left|\mathcal{D}_{0}\right|$ is bounded with high probability (w.h.p.).

Proposition 5. Let $\beta_{i}^{+}:=\sum_{j} \beta_{i j}$ and $\beta_{\max }^{+}=\max _{i}\left(\beta_{i}^{+}\right)$. If $\beta_{\max }^{+}<1$, then

$$
\mathbb{E}\left[\left|\mathcal{C}_{1}\right|\right] \leq \frac{1}{1-\beta_{\max }^{+}}\left|\mathcal{D}_{0}\right|,
$$

which in particular implies that $\left|\mathcal{C}_{1}\right| /\left|\mathcal{D}_{0}\right|=O_{p}(1)$.

The following example shows how the above proposition can be applied in the case where the out degrees of the financial network are bounded from the above.

Example 6 (Bounded out-degree graphs). Assume that the out degrees of the financial network are bounded from the above by $\Delta$ and $\beta_{i j}=\beta$ is constant over all existing links. The above proposition implies that if $\beta<\frac{1}{\Delta}$ then the network is resilient and we have for any finite set $\mathcal{D}_{0}, \mathbb{P}\left(\left|\mathcal{C}_{1}\right|>\omega(n)\right) \rightarrow 0$ for any $\omega(n) \rightarrow \infty$.

So far, we have given conditions for the size of $\mathcal{C}_{1}$ to be small in terms of the maximum row sum of contagion probability matrix. We now consider the $L_{2}$ norm of the matrix $B$. Let us denote by $\lambda_{\max }(B)=\|B\|_{2}$ the largest singular value of $B$ which is the square root of the largest eigenvalue of the positive-semidefinite matrix $B^{T} B$. The following proposition shows that the amplification $\left|\mathcal{C}_{1}\right| /\left|\mathcal{D}_{0}\right|$ is $O_{p}(\sqrt{n})$ whenever the largest singular value is smaller than 1. Intuitively, it is natural to characterize the subcritical regime in terms of the largest singular value, since this represents the rate of growth of the number of (directed) paths in the graph [26].

Proposition 7. If $\lambda_{\max }(B)<1$, then

$$
\mathbb{E}\left[\left|\mathcal{C}_{1}\right|\right] \leq \frac{1}{1-\lambda_{\max }(B)} \sqrt{n\left|\mathcal{D}_{0}\right|},
$$

which in particular implies that $\left|\mathcal{C}_{1}\right|=O_{p}\left(\sqrt{n\left|\mathcal{D}_{0}\right|}\right)$.

The following example shows that the upper bound in the above proposition is asymptotically tight in the case of star networks. Star networks are relevant to the post-crisis environment since a large part of the interbank liabilities are centrally cleared. As we introduce a central counterparty clearing (CCP) into the financial network (when all exposures are cleared), the financial network becomes a star network, see e.g. [9, 33]. 
Example 8 (Star networks). Consider a star network, consisting of a CCP denoted by node 1 and $n-1$ leaves. Assume the case where the CCP defaults, and $\mathcal{D}_{0}=\{1\}$. It is then clear that

$$
\mathbb{E}\left[\left|\mathcal{C}_{1}\right|\right]=1+\sum_{j=2}^{n} \beta_{1 j}
$$

Moreover, the largest singular value of $B$ satisfies (by the Cauchy-Schwarz inequality)

$$
\lambda_{\max }(B)=\sup _{\mathbf{x} \neq 0} \frac{\|B \mathbf{x}\|}{\|\mathbf{x}\|}=\sup _{\mathbf{x} \neq 0} \frac{\sum_{j=2}^{n} \beta_{1 j} x_{j}}{\sqrt{\sum_{j=2}^{n} x_{j}^{2}}}=\sqrt{\sum_{j=2}^{n} \beta_{1 j}^{2}} .
$$

So the above proposition says that if $\sum_{j=2}^{n} \beta_{1 j}^{2}<1$ then

$$
\lambda_{\max }(B)=\sqrt{\sum_{j=2}^{n} \beta_{1 j}^{2}} \geq 1-\frac{\sqrt{n}}{1+\sum_{j=2}^{n} \beta_{1 j}} .
$$

In particular suppose that $\beta_{i j}=\beta$ and $\lambda_{\max }(B)=\sqrt{\sum_{j=2}^{n} \beta_{1 j}^{2}}=\beta \sqrt{n-1}=\gamma<1$. Then we have

$$
\mathbb{E}\left[\left|\mathcal{C}_{1}\right|\right]=1+\sum_{j=2}^{n} \beta_{1 j}=1+\beta(n-1)=1+\gamma \sqrt{n-1}
$$

which is close to the upper bound $\frac{\sqrt{n}}{1-\gamma}$ given by above proposition. Moreover, in this case, using large deviation techniques for the binomial distributions (like Chernoff's inequality) we can show that for $\gamma^{\prime}<\gamma, \mathbb{P}\left(\left|\mathcal{C}_{1}\right| \leq \gamma^{\prime} \sqrt{n}\right)$ goes to 0 , exponentially in $n$, when $n$ goes to infinity.

Let us now define for $k=k(n)<n$,

$$
\eta(k):=\max _{S \subseteq[n], k \leq|S| \leq n} \frac{\beta^{+}\left(S, S^{c}\right)}{|S|},
$$

where $\beta^{+}\left(S, S^{c}\right)=\sum_{i \in S} \sum_{j \in S^{c}} \beta_{i j}$ and $S^{c}$ denotes the complement of the set $S$. The following proposition establishes another upper-bound on the size of the first order contagion. This upper bound is given by the size of a set that contains the fundamental set of defaults, but from which there is a small number of contagious links leading outside the set. The intuition behind this proposition is that if the average degree out of a set of size larger than $k$ is smaller than 1 (for all such sets) then the size of the first order cascade is $O_{p}(k)$.

Proposition 9. Assume that for some $k=k(n)=o(n)$ we have $\eta(k)<1$. Then

$$
\mathbb{E}\left[\left|\mathcal{C}_{1}\right|\right] \leq \frac{1}{1-\eta(k)} \max \left(k,\left|\mathcal{D}_{0}\right|\right)
$$

which in particular implies that $\left|\mathcal{C}_{1}\right|=O_{p}\left(\left|\mathcal{D}_{0}\right|+k(n)\right)$.

Example 10 (Erdős-Rényi random graphs). The (directed) Erdős-Rényi random graph $\operatorname{ER}(n, p)$ with parameters $n$ and $p$ consists of $n$ nodes, and each of the $n(n-1)$ possible links in a directed graph on $n$ labelled vertices is present independently with probability $p$. If $p=1$, then this is the complete graph. Assume that $\beta_{i j}=\beta$ for all $i, j \in[n], i \neq j$ (the general case will be discussed in Section 4). In this case, it is not hard to show that w.h.p. 
for $k(n) \gg \log n$ and for $c \geq \lim \sup _{n \rightarrow \infty}(n-1) p \beta$ we have $\eta(k) \leq c$. We thus conclude by the above proposition that if there exists some constant $c$ satisfying $\lim \sup _{n \rightarrow \infty}(n-1) p \beta \leq$ $c<1$ then, for any $\omega(n) \rightarrow \infty$ as $n \rightarrow \infty$ and a finite set $\mathcal{D}_{0}$, we have (as $n \rightarrow \infty$ )

$$
\mathbb{P}\left(\left|\mathcal{C}_{1}\right|>\omega(n) \log n\right) \rightarrow 0 .
$$

\section{Inhomogeneous random financial networks}

So far, we have considered the financial network as given and that the randomness stems only from the recovery rates. In this section we assume that we have only partial information about the financial network. In particular, the matrix $L_{i j}$ is not observable. We assume that the financial institutions have different types which are in a certain type space $\mathcal{S}$ and we only have information about their types. The set of types can be large enough to classify the banks according to the available information. We model the financial network as a random network consistent with the observation of types. The probability that there is a contagious link between any two banks depends thus on the types of these two banks.

When there are banks of just 2 types, e.g., core and periphery as in Example 13, with banks that are homogenous within each class, then it suffices to take $\mathcal{S}=\left\{s_{1}, s_{2}\right\}$. However, the model allows for rather general sets of types of the financial institutions, both finite as well as (countably or uncountably) infinite. An example of an uncountably infinite type space could be types related to the total liability $L_{i}$ and the total asset $A_{i}$ of each individual bank $i$ in the financial network; see Example 14.

Assume we know how many institutions there are of a given type. This is described in terms of a measure $\mu$, where for $A \subseteq \mathcal{S}, \mu(A)$ denotes the proportion of banks having a type in $A$. Since only the types of the banks are observable and not their linkages, it is natural to assume in the partial information setting that the probability $\beta_{i j}$ of having a contagious link from $i$ to $j$ depends only on the types of $i$ and $j$. Namely (in our general model) we consider a kernel

$$
\hat{\beta}=\hat{\beta}^{(n)}: \mathcal{S}^{2} \rightarrow[0, \infty),
$$

where for $s, s^{\prime} \in \mathcal{S}, \hat{\beta}\left(s, s^{\prime}\right) / n \wedge 1$ is the probability of having a contagious link from an institution of type $s$ to an institution of type $s^{\prime}$. As a (natural) technical assumption we consider in the following that

$$
\sup _{s, s^{\prime}, n} \hat{\beta}\left(s, s^{\prime}\right)<\infty .
$$

When $\mathcal{S}=\left\{s_{1}\right\}$ we have $\beta_{i j}=\beta$ and we find as a special case the Erdös-Rényi random graph which was studied in Example 10. The inhomogenous model of random graphs was introduced in $[47,21]$. The directed inhomogeneous random graphs was studied in [17].

\subsection{The finite-type case}

We first treat the case when the set of types is finite. Namely, fix $r \geq 2$ and suppose we have a financial network with $r$ different types. Let $\mathcal{S}=\left\{s_{1}, s_{2}, \ldots, s_{r}\right\}$ and let $s(v)$ denote the type of a bank $v \in[n]$. Let $n_{i}$ denote the number of vertices of type $s_{i}$, i.e.,

$$
n_{i}:=\#\left\{v \in[n] \mid s(v)=s_{i}\right\},
$$


so that $n_{1}+n_{2}+\cdots+n_{r}=n$ and let $\mu^{(n)}\left(s_{i}\right)=n_{i} / n$. We shall assume that the fraction of vertices of a given type is asymptotically positive constant which means there is a probability distribution $\mu$ (independent of $n$ ) such that

$$
\lim _{n \rightarrow \infty} \mu^{(n)}\left(s_{i}\right)=\mu_{i},
$$

so that we have $\mu_{i}>0$ and $n_{i}-\mu_{i} n=o(n)$. We then assume that the probability $\beta_{u v}$ of having a contagious link from bank $u$ to bank $v$ depends only on the types of $u$ and $v$ so that

$$
\beta_{u v}=\hat{\beta}(s(u), s(v)) / n .
$$

Consider now the $r \times r$ matrix $\bar{B}=\left(\bar{b}_{i j}\right)_{1 \leq i, j \leq r}$ where

$$
\bar{b}_{i j}:=\mu_{j} \hat{\beta}\left(s_{i}, s_{j}\right)
$$

is the average number of contagious links from an institution of type $s_{i}$ to the institutions of type $s_{j}$. The following theorem says that if the largest singular value of the contagion probability matrix $\bar{B}$ is smaller than one, then the amplification $\left|\mathcal{C}_{1}\right| /\left|\mathcal{D}_{0}\right|$ is $O_{p}(\log n)$. On the contrary, if the largest singular value of the matrix $\bar{B}$ is greater than one, then there exists a (giant) component strongly connected by contagious links which represents a positive faction of the financial system. This marks a phase transition in the size of the first order cascade. In the supercritical regime, if any of the initially defaulted nodes belongs to the component strongly connected by contagious links, then this whole component will default, and thus the final cascade will be a positive fraction of the financial network.

Theorem 11. The following holds:

- If $\lambda_{\max }(\bar{B})<1$ then $\left|\mathcal{C}_{1}\right| /\left|\mathcal{D}_{0}\right|=O_{p}(\log n)$;

- If $\lambda_{\max }(\bar{B})>1$ then w.h.p. there exists a strongly connected set of nodes representing a positive fraction of the financial system such that the default of any node belonging to this set can trigger the default of all nodes in the set.

To give the intuition behind the above theorem, in the following, we describe an approximation of the local structure of the inhomogeneous financial network (induced by contagious links) by a multi-type branching process.

Remark 12 (Branching process approximation). Consider the multi-type Galton-Watson processes where the particles are of types from $\mathcal{S}=\left\{s_{1}, s_{2}, \ldots, s_{r}\right\}$. Given $i \in[r]$ let $\mathcal{X}_{i}^{+}$ (resp. $\mathcal{X}_{i}^{-}$) denote the Galton-Watson process starting at a particle of type $s_{i}$ such that the number of children of type $s_{k} \in \mathcal{S}$ of a particle of type $s_{j} \in \mathcal{S}$ has a Poisson distribution with mean $\hat{\beta}\left(s_{j}, s_{k}\right) \mu_{k}$ (resp. $\left.\hat{\beta}\left(s_{k}, s_{j}\right) \mu_{k}\right)$, for all $1 \leq j, k \leq r$. Let $\xi_{i}^{+}$denote the survival probability of $\mathcal{X}_{i}^{+}$. Thus the vector of survival probabilities $\left(\xi_{1}^{+}, \ldots, \xi_{1}^{+}\right)$is the maximal fixed point in $[0,1]$ of the following fixed point equation; see e.g., [15]:

$$
\xi_{i}^{+}=1-\mathbb{E}\left(-\sum_{j=1}^{r} \mu_{j} \hat{\beta}\left(s_{i}, s_{j}\right) \xi_{j}^{+}\right),
$$

for all $i=1, \ldots, r$. Respectively $\xi_{i}^{-}$denotes the survival probability of $\mathcal{X}_{i}^{-}$and $\left(\xi_{1}^{-}, \ldots, \xi_{1}^{-}\right)$ is the maximal fixed point in $[0,1]$ of

$$
\xi_{i}^{-}=1-\mathbb{E}\left(-\sum_{j=1}^{r} \mu_{j} \hat{\beta}\left(s_{j}, s_{i}\right) \xi_{j}^{-}\right),
$$


for all $i=1, \ldots, r$. We then let $\xi^{*}=\sum_{i=1}^{r} \xi_{i}^{+} \xi_{i}^{-}$. The main result of [17] is that if $\bar{B}$ is an irreducible matrix then the size of the strongly connected giant component in finite-type inhomogeneous random graphs is given by $\xi^{*} n+o(n)$ w.h.p..

In the following example, we consider the particular case of a core-periphery economy. Recent empirical evidence suggests that financial networks exhibit a core-periphery network structure, the core being highly interconnected and peripheral banks having few connections only with banks in the core, see e.g., [30]. The previous theorem gives in this case a simple and testable condition for the network to be resilient.

Example 13 (Core-periphery structure). Suppose that we have a financial network in which there are $n_{C}$ banks of type $C$ or "core" and $n_{P}$ banks of type $P$ or "periphery" with $n_{C}+n_{P}=n, n_{C} / n \rightarrow \mu_{C}$ and $n_{P} / n \rightarrow \mu_{P}$ as $n \rightarrow \infty$. Suppose that there are no (contagious) links between any two periphery banks. Then the above theorem implies that if

$$
\mu_{C} \mu_{P} \hat{\beta}(C, P) \hat{\beta}(P, C)+\mu_{C} \hat{\beta}(C, C)<1,
$$

then the size of the first order cascade is small and we have $\left|\mathcal{C}_{1}\right| /\left|\mathcal{D}_{0}\right|=O_{p}(\log n)$.

\subsection{The general case}

In this section we extend the result of Theorem 11 to a much more general setting where the type space $\mathcal{S}$ is a separable metric space. For $A \subseteq \mathcal{S}, \mu^{(n)}(A)$ denotes the proportion of banks having a type in $A$. Writing $s_{i}=s_{i}^{(n)}$ for the type of bank $i \in[n]$, and $\delta_{s}$ for the measure consisting of a point mass of weight 1 at $s$, and

$$
\mu^{(n)}:=\frac{1}{n} \sum_{i=1}^{n} \delta_{s_{i}}
$$

for the empirical distribution of the types of banks, we shall assume that $\mu^{(n)}$ converges (in probability in case that $s_{i}$ are random) to $\mu$ as $n \rightarrow \infty$. The convergence condition $\mu^{(n)} \rightarrow \mu$ means exactly that for every $\mu$-continuity set $A$,

$$
\mu^{(n)}(A):=\#\left\{i: s_{i}^{(n)} \in A\right\} / n \rightarrow \mu(A) .
$$

Example 14. An example of an uncountably infinite type space is given when the types represent the size of the total interbank assets $A_{i}$, the size of total interbank liabilities $L_{i}$ and the capital $C_{i}$ of each individual bank $i$ in the financial network. We now let the measurable space of types $\mathcal{S}:=\left([0, \infty)^{3}, \mathcal{B}\left([0, \infty)^{3}\right)\right)$, endowed with the Borel sigma-algebra. A type $(x, y, z) \in \mathcal{S}$ is interpreted as the size of interbank assets, interbank liabilities and capital of a bank that has this type. For any $n$ and sequence of assets, liabilities and capital $\left(A_{i}^{(n)}, L_{i}^{(n)}, C_{i}^{(n)}\right)_{i \in[n]}$, we write

$$
F^{(n)}(x, y, z):=\#\left\{i \in[n], A_{i}^{(n)} \leq x, L_{i}^{(n)} \leq y, C_{i}^{(n)} \leq z\right\} / n .
$$

We then need to assume that $F^{(n)}$ converges weakly as the size of the network tends to $\infty$, i.e, there is a distribution function $F:[0, \infty)^{3} \rightarrow[0,1]$ such that for all $(x, y, z)$ at which $F$ is continuous $\lim _{n \rightarrow \infty} F^{(n)}(x, y, z)=F(x, y, z)$.

We shall assume that the probability $\beta_{i j}$ of having a contagious link from $i$ to $j$ depends only on the types of $i$ and $j$. Namely (in our general model) we consider a kernel

$$
\hat{\beta}=\hat{\beta}^{(n)}: \mathcal{S}^{2} \rightarrow[0, \infty),
$$


where for $i, j \in[n]$, the probability of having a contagious link from $i$ with type $s_{i}$ to $j$ with type $s_{j}$ is given by $\hat{\beta}\left(s_{i}, s_{j}\right) / n$ independently of all other pairs $i, j$.

We say that a kernel $\hat{\beta}$ is graphical on a vertex space $\mathcal{V}=\left(\mathcal{S}, \mu,\left(s_{1}, \ldots, s_{n}\right)\right)$ if the following conditions hold:

(i) $\hat{\beta}$ is continuous on $\mathcal{S} \times \mathcal{S}$;

(ii) $\hat{\beta} \in \mathcal{L}^{1}(\mathcal{S} \times \mathcal{S})$;

(iii) $\frac{1}{n} \sum_{i, j \in[n]} \hat{\beta}\left(s_{i}, s_{j}\right) \longrightarrow \int_{\mathcal{S}} \int_{\mathcal{S}} \hat{\beta}\left(s, s^{\prime}\right) \mu(d s) \mu\left(d s^{\prime}\right)$.

We assume that $\hat{\beta}$ is graphical in the following. Similar to the case of non directed inhomogeneous random graphs, see [21], the number of outgoing (resp. incoming) contagious links belonging to a vertex of a given type $x$ is asymptotically distributed as a Poisson random variable with a mean $\lambda^{+}(x)$ (reps. $\lambda^{-}(x)$ ) where

$$
\lambda^{+}(x):=\int_{\mathcal{S}} \hat{\beta}(x, y) \mu(d y) \text { and } \lambda^{-}(x):=\int_{\mathcal{S}} \hat{\beta}(y, x) \mu(d y) .
$$

Given a kernel $\hat{\beta}$ on a ground space $(\mathcal{S}, \mu)$, let $T_{\hat{\beta}}^{+}$and $T_{\hat{\beta}}^{-}$be the integral operators on $(\mathcal{S}, \mu)$ with kernel $\hat{\beta}$, defined by

$$
\begin{aligned}
\left(T_{\hat{\beta}}^{+} f\right)(x) & :=\int_{\mathcal{S}} \hat{\beta}(x, y) f(y) \mu(d y), \\
\left(T_{\hat{\beta}}^{-} f\right)(x) & :=\int_{\mathcal{S}} \hat{\beta}(y, x) f(y) \mu(d y),
\end{aligned}
$$

for any (measurable) function $f$ such that this integral is defined (finite or $+\infty$ ) for almost every (a.e.) $x$. Note that if $\hat{\beta} \in \mathcal{L}^{1}(\mathcal{S} \times \mathcal{S})$, as we shall assume throughout, then $T_{\hat{\beta}}^{ \pm} f$ is also defined for every bounded $f$; in this case $T_{\hat{\beta}}^{ \pm} f \in \mathcal{L}^{1}(\mathcal{S})$ and thus $T_{\hat{\beta}}^{ \pm} f$ is finite a.e.

We define

$$
\left\|T_{\hat{\beta}}^{+}\right\|:=\sup \left\{\left\|T_{\hat{\beta}}^{+} f\right\|_{2}: f \geq 0,\|f\|_{2} \leq 1\right\} \leq \infty .
$$

The following theorem shows that, in the general case, the resilience of the financial network can be characterized using the norm of the operator $T_{\hat{\beta}}^{+}$.

Theorem 15. The following holds:

- If ||$T_{\hat{\beta}}^{+}||<1$ then $\left|\mathcal{C}_{1}\right| /\left|\mathcal{D}_{0}\right|=O_{p}(\log n)$;

- If $\left\|T_{\hat{\beta}}^{+}\right\|>1$ then with high probability, there exists a strongly connected set of nodes representing a positive fraction of the financial system such that the default of any node belonging to this set can trigger the default of all nodes in the set.

Similarly to the finite-type case, we can give the intuition behind the above theorem by looking at the following multi-type branching process. The survival probability for the multi-type branching process is determined in terms of the operator $1-\exp \left(-T_{\hat{\beta}}^{+} f\right)$.

Remark 16 (Branching process approximation). Consider the multi-type Galton-Watson branching process $\mathcal{X}_{s}^{+}\left(\right.$reps. $\left.\mathcal{X}_{s}^{-}\right)$associated to $(\mathcal{S}, \mu, \hat{\beta})$. This starts with a single particle whose type is $s$. Each particle of type $x$ has a set of children whose types are distributed 
as a Poisson process on $\mathcal{S}$ with intensity $\hat{\beta}(x, y) d \mu(y)$ (resp. $\hat{\beta}(y, x) d \mu(y))$. In other words, the number of children with types in a subset $A \subseteq \mathcal{S}$ has a Poisson distribution with mean $\int_{A} \hat{\beta}(x, y) d \mu(y)$, and these numbers are independent for disjoint sets $A$ and for different particles; see [21]. Let $\xi^{+}(s)$ (reps. $\xi^{-}(s)$ ) be the survival probabilitiy for the branching process $\mathcal{X}_{s}^{+}\left(\right.$reps. $\left.\mathcal{X}_{s}^{-}\right)$. We define the operators $\Phi_{\hat{\beta}}^{+}$and $\Phi_{\hat{\beta}}^{-}$by

$$
\Phi_{\hat{\beta}}^{ \pm} f=1-\exp \left(-T_{\hat{\beta}}^{ \pm} f\right),
$$

for $f \geq 0$. Note that for such $f$ we have $0 \leq T_{\hat{\beta}}^{ \pm} f \leq \infty$, and thus $0 \leq \Phi_{\hat{\beta}}^{ \pm} f \leq 1$. The survival probabilities $\xi^{+}(s)$ and $\xi^{-}(s)$ are the maximal fixed point of the non-linear operators $\Phi_{\hat{\beta}}^{+}$ and $\Phi_{\hat{\beta}}^{-}$respectively; see [21]. We then set

$$
\xi^{*}=\int_{\mathcal{S}} \xi^{+}(s) \xi^{-}(s) \mu(d s) .
$$

The results of $[21,17]$ show that the size of the strongly connected giant component in (general) inhomogeneous random graphs is given by $\xi^{*} n+o(n)$ with high probability if the kernel $\hat{\beta}$ on a ground space $(\mathcal{S}, \mu)$ is irreducible; a kernel $\hat{\beta}$ on a ground space $(\mathcal{S}, \mu)$ is reducible if there exists $A \subset \mathcal{S}$ with $0<\mu(A)<1$ such that $\hat{\beta}=0$ a.e. on $A \times(\mathcal{S} / A)$, and irreducible otherwise.

\section{$5 \quad$ Final size of default cascades in large networks}

In the previous section we characterized the size of the first-order contagion $\left|\mathcal{C}_{1}\right|$ when the skeleton of contagious links is drawn from an inhomogenous random graph. We now turn our attention to the final set of defaults $\mathcal{D}_{n-1}$ and we characterize its size.

In order to compare our results with previous findings, we first summarize the results in [8], which give limit theorems on the final size of the default cascade. These results hold in an information setting where the distribution of the degree and default threshold of the banks is known. More importantly, these results are now stated under a relaxed condition on the second order moment of the degree distribution; the distribution of degrees may now have infinite variance, as is the case in many real-world networks.

We then consider the case of power-law degree distribution of financial institutions and state a theorem which shows that if the size of $\mathcal{C}_{1}$ (and the number of outgoing links from $\mathcal{C}_{1}$ to the rest of the network) is smaller than some critical parameter then the final size of cascade will be small.

\subsection{Limit theorems}

Similarly as in the previous section, we assume that we have only partial information about the financial network and in particular on the matrix $L_{i j}$. Namely we assume that for each institution $i \in[n]$, we are given the number of its creditors denoted by

$$
d_{\text {out }}^{(n)}(i):=\#\left\{j \in[n]: L_{i j}>0\right\},
$$

and the number of its debtors denoted by

$$
d_{\mathrm{in}}^{(n)}(i):=\#\left\{j \in[n]: L_{j i}>0\right\} .
$$


The empirical distribution of the degrees is denoted by $\mu^{(n)}$ which is assumed to converge to some probability distribution $\mu$ with finite average $\lambda$ as $n \rightarrow \infty$; (for all $j, k \in \mathbb{N}$ )

$$
\mu^{(n)}(j, k):=\#\left\{i \in[n] \mid d_{\text {in }}^{(n)}(i)=j, d_{\text {out }}^{(n)}(i)=k\right\} \rightarrow \mu(j, k),
$$

and,

$$
\lambda:=\sum_{j, k} j \mu(j, k)=\sum_{j, k} k \mu(j, k) \in(0, \infty)
$$

Moreover, we need to assume that (as $n \rightarrow \infty$ )

$$
\sum_{j, k} j \mu^{(n)}(j, k)=\sum_{j, k} k \mu^{(n)}(j, k) \rightarrow \lambda .
$$

Assume now that for each institution $i \in[n]$, we are given the set of non-zero elements of the $i$-th column of the liability matrix $L$ which is given by

$$
L_{\text {in }}(i)=\left\{L_{j i} \mid L_{j i}>0\right\} .
$$

We then consider the random financial network which captures this data for each node and is constructed by configuration model: associate to each node $i$, two sets: $H_{\text {out }}(i)$ representing its out-going half-edges, and $H_{\text {in }}(i)$ representing its in-coming half-edges, with $\left|H_{\text {out }}(i)\right|=d_{\text {out }}^{(n)}(i)$, and $\left|H_{\text {in }}(i)\right|=d_{\text {in }}^{(n)}(i)$. Moreover the elements in the set $L_{\text {in }}(i)$ are assigned (randomly) to each incoming half-edges. Let $H_{\text {out }}=\bigcup_{i \in[n]} H_{\text {out }}(i)$ and $H_{\text {in }}=$ $\bigcup_{i} H_{\text {in }}(i)$. A configuration is a matching of $H_{\text {out }}$ with $H_{\text {in }}$. When an out-going half-edge of node $i$ is matched with an in-coming half-edge of node $j$, a directed edge from $i$ to $j$ appears in the graph. The configuration model is the random directed multigraph which is uniformly distributed across all configurations. Observe that the self-loops may occur, these become rare as $n \rightarrow \infty$. It is easy to see conditional on the multigraph being simple graph, we obtain a uniformly distributed random graph with these given degree sequences; see $[8]$.

The sequences of continuous liabilities, external assets, deposits and external shocks are mapped into discrete sequences representing the default threshold for each institution, with the result of this mapping represented by the quantities $p^{(n)}(j, k, \theta)$. These are assumed to converge to some distribution

$$
p^{(n)}(j, k, \theta) \rightarrow p(j, k, \theta),
$$

where the limit distribution can be interpreted as the fraction of banks with degree $(j, k)$ that default exactly after $\theta$ debtor defaults; see [8] for more details.

Remark 17. Under the assumption that $R_{i j}$ are i.i.d. with support on $[\underline{R}, \bar{R}]$ for all $i, j$, it is easy to see that the number of contagious links of each node can be drawn independently of the matching in the configuration model. Indeed, when the set $L_{\text {in }}(i)$ is assigned to the incoming half-edges, the number of contagious incoming half-edges of bank $i$ is given by a random variable

$$
N_{i}:=\sum_{\ell_{k} \in L_{\text {in }}(i)} \mathbb{1}_{\left(1-R_{k}\right) \ell_{k}>C(i)},
$$

with $R_{k}$ a random variable with value in $(\underline{R}, \bar{R})$, with the same distribution as all recovery rates $R_{i j}$. We then have (for large $n$ )

$$
\beta_{i j} \approx \frac{d_{\text {out }}(i)}{\sum_{k=1}^{n} d_{\text {out }}(k)} \mathbb{E}\left(N_{j}\right) \approx \frac{d_{\text {out }}(i) d_{\text {in }}(j)}{\sum_{k=1}^{n} d_{\text {out }}(k)} p\left(d_{\text {in }}(j), d_{\text {out }}(j), 1\right) .
$$


We define the function $I:[0,1] \rightarrow[0,1]$ as

$$
I(\pi):=\sum_{j, k, \theta} \frac{k \mu(j, k)}{\lambda} p(j, k, \theta) \mathbb{P}(\operatorname{Bin}(j, \pi) \geq \theta),
$$

and let $\pi^{*}$ be the smallest fixed point of $I$ in $[0,1]$.

The following theorem proved in [8] gives the asymptotic size of contagion. In order to ensure that with positive probability the graph is simple in the limit, we assumed in [8] a condition of finite second order moment for the degree distribution. However, the proof in [8] for the following two theorems does not require this condition, so here it is relaxed.

Theorem 18. We have:

- If $\pi^{*}=1$ then asymptotically all nodes default during the cascades:

$$
\frac{\left|\mathcal{D}_{n-1}\right|}{n} \stackrel{p}{\longrightarrow} 1
$$

- If $\pi^{*}<1$ and furthermore $\pi^{*}$ is a stable fixed point of $I\left(I^{\prime}\left(\pi^{*}\right) \neq 1\right)$, then

$$
\frac{\left|\mathcal{D}_{n-1}\right|}{n} \stackrel{p}{\longrightarrow} J\left(\pi^{*}\right):=\sum_{j, k, \theta} \mu(j, k) p(j, k, \theta) \mathbb{P}\left(\operatorname{Bin}\left(j, \pi^{*}\right) \geq \theta\right) .
$$

We define as the resilience measure the following function of the network's features, which takes values in $(-\infty, 1]$ :

$$
1-\sum_{j, k} \frac{j k}{\lambda} \mu(j, k) p(j, k, 1)
$$

The second theorem of [8] is the following.

Theorem 19. The response of the financial network to the initial default of a small number of nodes depends on its intrinsic frailty in the following way:

- If the resilience measure is positive and moreover $\sum_{j, k} j k \mu(j, k)<\infty$, then w.h.p. for any finite set $\mathcal{D}_{0}$, the final fraction of defaults is negligible.

- If the resilience measure is negative, then there exists a strongly connected set of nodes representing a positive fraction of the financial system such that, w.h.p. any node belonging to this set can trigger the default of all nodes in the set.

We now suppose that the resilience condition given by above theorem is satisfied so that the resilience measure is positive and we have $\sum_{j, k} \frac{j k}{\lambda} \mu(j, k) p(j, k, 1)<1$, and assume that only a small fraction $\epsilon$ of all nodes represent fundamental defaults, i.e., $p(j, k, 0)=\epsilon$ for all $j, k \in[n]$. As a corollary of Theorem 18 we have:

Corollary 20. Assume that the resilience condition is satisfied and moreover we have $\sum_{j, k} j k \mu(j, k)<\infty$. When a small fraction $\epsilon$ of all nodes represent fundamental defaults, we have

$$
\frac{\left|\mathcal{D}_{n-1}\right|}{n} \stackrel{p}{\longrightarrow} \epsilon \underbrace{\left(1+\frac{\sum_{j, k} j \mu(j, k) p(j, k, 1)}{1-\sum_{j, k} \frac{\mu(j, k) j k}{\lambda} p(j, k, 1)}\right.}_{\text {Amplification }})+o(\epsilon) .
$$


Note that the above corollary requires that $\sum_{j, k} j k \mu(j, k)<\infty$. In [8] this is implied by the condition of finite second order moment for the degree distribution. Thus, under the resilience condition, assuming that $\sum_{j, k} j k \mu(j, k)<\infty$, gives a finite amplification of defaults given by (18). On the other hand, if $\sum_{j, k} j k \mu(j, k)=\infty$ (as is the case for many real-world networks) then the resilience condition is still a necessary condition for the resilience of the financial network. In this case, as we show below, a positive small fraction of initial defaults may still trigger a large cascade under certain conditions.

This is the object of a new result of this paper that we state below. Consider the case when $\sum_{j, k} j k \mu(j, k)=\infty$ and suppose there is a fraction of banks with a finite threshold to default and at the same time with a large number of creditors and debtors. Then these banks will amplify the initial defaults: Their large number of incoming links will likely be connected to multiple initially defaulted banks and thus these banks reach their default threshold. Moreover, these banks have at the same time a large out-degree and thus they will increase the rate of the epidemics' spread.

Corollary 21. Assume that for some $\Theta \in \mathbb{N},, \gamma \in \mathbb{R}^{+}$and $\beta \in(2,3)$ :

$$
\sum_{k} \sum_{\theta=1}^{\Theta} k \mu(j, k) p(j, k, \theta) \geq \gamma j^{-\beta+1}
$$

for all $j \in \mathbb{N}$. Then there exists $\hat{\pi}>0$ the smallest positive solution of

$$
\pi=\sum_{j, k} \sum_{\theta \geq 1} \frac{k \mu(j, k)}{\lambda} p(j, k, \theta) \mathbb{P}(\operatorname{Bin}(j, \pi) \geq \theta)
$$

such that, for $\epsilon$ small enough and $n$ large enough, the final fraction of defaults is given by $J(\hat{\pi})>0$. Namely, for all $\delta>0$ there exists $n(\delta)$ and $\epsilon(\delta)$ such that for all $n>n(\delta)$ and $\epsilon<\epsilon(\delta)$ we have

$$
\mathbb{P}\left(\left|\frac{\left|\mathcal{D}_{n-1}\right|}{n}-J(\hat{\pi})\right|<\delta\right)>1-\delta .
$$

The above corollary shows a jump discontinuity at 0 for the final size of the cascade when $\sum_{k} \sum_{\theta=1}^{\Theta} k \mu(j, k) p(j, k, \theta) \geq \gamma j^{-\beta+1}$ for all $j \in \mathbb{N}$. In particular this condition implies that (since $\beta \in(2,3)$ )

$$
\sum_{j, k} j k \mu(j, k) \geq \gamma \sum_{j} j^{-\beta+2}=\infty .
$$

Of course, the interest of this result is the case $\Theta>1$, since when $\Theta=1$ we already know with this condition the network is not resilient. Note that in the fixed point equation (19), the threshold runs over $\theta \geq 1$ contrary to the fixed point of the function $I$ in Theorem 18. Corollary 21 states that as the fraction of banks with $\theta=0$ tends to zero, the number of banks that default (which have a threshold $\theta>0$ ) represents a positive fraction of the system. We also give a precise value for this final fraction of defaults.

Given that a small fraction of defaults may lead to a large number of defaults, the natural question is to give sufficient conditions on the number of initial defaults and the set of banks touched by the first order cascade such that the fraction of final defaults is small, i.e., $o(n)$. In the next section, we give such a condition for the case of power law random graphs with parameter $\beta \in(2,3)$, whose second moment of the degree distribution diverges. 


\subsection{Power-law random graphs}

This section focuses on the special case of degree distribution of financial institutions, namely those following a power law distribution. In particular, we consider a random graph model that is asymptotically equivalent to a model considered by Chung and $\mathrm{Lu}$ [26], and is a special case of the inhomogeneous random graph model studied in the previous section.

In order to define the model we consider for any $n \in \mathbb{N}$ the vertex set $[n]$. Each vertex $i$ is assigned two positive weights $\bar{d}_{\text {out }}^{(n)}(i)$ and $\bar{d}_{\text {in }}^{(n)}(i)$ which represent the expected out degree and the expected in degree of each institution $i \in[n]$. We assume that $\sum_{i=1}^{n} \bar{d}_{\text {out }}^{(n)}(i)=$ $\sum_{i=1}^{n} \bar{d}_{\text {in }}^{(n)}(i)$ and we set

$$
W_{n}=\sum_{i=1}^{n} \bar{d}_{\mathrm{out}}^{(n)}(i)=\sum_{i=1}^{n} \bar{d}_{\mathrm{in}}^{(n)}(i) .
$$

In our random graph model, the event of including the link $\{i \rightarrow j\}$ in the resulting graph is independent of the inclusion of any other links, and its probability equals

$$
p_{i \rightarrow j}=\min \left\{\frac{\bar{d}_{\text {out }}(i) \bar{d}_{\text {in }}(j)}{W_{n}}, 1\right\} .
$$

The degree sequence of the resulting graph follows a power law with high probability, provided that the sequence of weights follows a power law (see [48] for a detailed discussion). Such random graphs are also characterized as ultra-small worlds, due to the fact that the typical distance of two vertices that belong to the same component is $O(\log \log n)$; see e.g., [26] or [48].

For any $n \in \mathbb{N}$ and any sequence of expected degrees $\mathbf{d}=\mathbf{d}(n)$ we write

$$
F^{(n)}(x, y)=n^{-1} \sum_{i=1}^{n} \mathbf{1}\left[\bar{d}_{\text {in }}(i) \leq x, \bar{d}_{\text {out }}(i) \leq y\right], \quad \forall x, y \in[0, \infty)
$$

for the empirical distribution function of the expected degrees of a vertex chosen uniformly at random.

We will assume that $F_{n}$ satisfies the following two conditions:

- There is a distribution function $F:[0, \infty) \times[0, \infty) \rightarrow[0,1]$ such that for all $x, y$ at which $F$ is continuous $\lim _{n \rightarrow \infty} F^{(n)}(x, y)=F(x, y)$;

- Let $\left(D_{\text {in }}^{(n)}, D_{\text {out }}^{(n)}\right)$ be random variables with distribution function $F^{(n)}$, and let $\left(D_{\text {in }}, D_{\text {out }}\right)$ be random variables with distribution function $F$, we have

$$
\lim _{n \rightarrow \infty} \mathbb{E}\left[D_{\text {in }}^{(n)}\right]=\lim _{n \rightarrow \infty} \mathbb{E}\left[D_{\text {out }}^{(n)}\right]=\mathbb{E}\left[D_{\text {in }}\right]=\mathbb{E}\left[D_{\text {out }}\right]=: \lambda .
$$

These conditions guarantee two important properties. First, the expected degree of a random vertex is approximately distributed as a random variable that follows a certain distribution. Second, this variable has finite mean and therefore the resulting graph has bounded average degree.

We define the empirical distribution of the expected in degrees as

$$
F_{\text {in }}^{(n)}(x)=n^{-1} \sum_{i=1}^{n} \mathbf{1}\left[\bar{d}_{\text {in }}(i) \leq x\right], \quad \forall x \in[0, \infty) .
$$


We now assume that $\left(\bar{d}_{\text {in }}(i)\right)_{i \in[n]}$ follows a power law distribution with parameter $\beta \in(2,3)$. This is described by the following condition:

There are $0<\gamma_{1}<\gamma_{2}, x_{0}>0$ and $0<\zeta$ such that for all $x_{0} \leq x \leq n^{\zeta}$

$$
\gamma_{1} x^{-\beta+1} \leq 1-F_{\text {in }}^{(n)}(x) \leq \gamma_{2} x^{-\beta+1},
$$

and $F_{\text {in }}^{(n)}(x)=0$ for $x<x_{0}$, but $F_{\text {in }}^{(n)}(x)=1$ for $x>n^{\zeta}$.

Note that according to above definition, for $\zeta>1 /(\beta-1)$, we have $n\left(1-F_{\text {in }}^{(n)}\left(n^{\zeta}\right)\right)=0$, since $1-F_{\text {in }}^{(n)}\left(n^{\zeta}\right) \leq \gamma_{2} n^{-\zeta(\beta-1)}=o\left(n^{-1}\right)$. Thus we assume $\zeta \leq 1 /(\beta-1)$ in the following.

The main result of this section is the following theorem which shows that if the size of $\mathcal{C}_{1}$ is sufficiently small, then the contagion process will not go beyond $\mathcal{C}_{1}$. In other words it is sufficient to understand a topological property of the network, namely the size of the set $\mathcal{C}_{1}$, to understand its cascading behavior.

Theorem 22. If $\sum_{i \in \mathcal{C}_{1}} \bar{d}_{\text {out }}(i) \ll d_{c}(n):=n^{\frac{1-\zeta(3-\beta)}{2}}$ then w.h.p. $\mathcal{D}_{n-1}=\mathcal{C}_{1}$.

Note that since $\zeta \leq 1 /(\beta-1)$ we have $d_{c}(n) \geq n^{\frac{\beta-2}{\beta-1}}$ and, in particular, the above theorem implies that if $\sum_{i \in \mathcal{C}_{1}} \bar{d}_{\text {out }}(i) \ll n^{\frac{\beta-2}{\beta-1}}$ then w.h.p. $\mathcal{D}_{n-1}=\mathcal{C}_{1}$.

Remark 23. Under some conditions on the degrees and threshold to default of the banks, the above condition becomes necessary for the resilience of financial network. Assume that there exists $\theta^{*} \in \mathbb{N}$ (large enough) and $\epsilon>0$ (small enough) such that any bank in the network defaults with probability at least $\epsilon$ when $\theta^{*}$ of its creditors (regardless which ones) are in default. Assume moreover that the out degrees and in degrees are highly correlated. Namely, there exists $K \in \mathbb{N}$ such that for all $i \in[n]$ :

$$
\frac{1}{K} \bar{d}_{\text {in }}(i) \leq \bar{d}_{\text {out }}(i) \leq K \bar{d}_{\text {in }}(i)
$$

Then the result of [10] on bootstrap percolation in power law random graphs implies that for $\zeta>\frac{\theta^{*}-1}{2 \theta^{*}-\beta+1}$, a positive fraction of banks will default w.h.p, i.e., there exists $\alpha>0$ such that w.h.p. $\left|\mathcal{D}_{n-1}\right|>\alpha n$, whenever

$$
\left|\mathcal{C}_{1}\right| \gg n^{\frac{\theta^{*}(1-\zeta)+\zeta(\beta-1)-1}{\theta^{*}}} .
$$

In particular when $\theta^{*}=2$ or $\zeta=1 /(\beta-1)$ we find that if $\left|\mathcal{C}_{1}\right| \gg d_{c}(n)$ then (w.h.p.) a positive fraction of banks will default so that the above condition is necessary.

\section{Conclusion}

In this paper we have proposed different frameworks for testing the possibility of large cascades in financial networks. Our model relies on specifying the first order contagion probabilities. Contagion may occur due to multiple channels, such as balance-sheet insolvency contagion, cash-flow insolvency contagion or highly relevant, contagion due to reliance on the same sources of unstable funding.

Our framework allows for the following specifications of the first order contagion probabilities. Under full observation of the interbank network, the uncertainty comes from the 
recovery rates. In case of insolvency contagion, the contagion probabilities are specified for any pair of banks using a model for the future recovery rates. Under partial observation of the interbank network, the linkages are unobservable and only certain characteristics of the banks are observable, such as capital, aggregate assets, total debt, short term debt, etc. We assume that for any pair of banks, the first-order contagion probability depends on these banks only through their characteristics. Given these probabilities, we derive testable conditions for large cascades.

Our results can be used in a regulatory risk management framework: a (random) financial network is acceptable if it does not allow for large cascades for a set of stress scenarios in which certain characteristics, such as capital or liquidity reserves are stressed. Moreover, our results point to the need to monitor the first order contagion probabilities, and specify capital and liquidity requirements in relation to these probabilities so that the financial network is acceptable.

\section{References}

[1] D. Acemoglu, V. M. Carvalho, A. Ozdaglar, and A. Tahbaz-Salehi. The network origins of aggregate fluctuations. Econometrica, 80(5):1977-2016, 2012.

[2] D. Acemoglu, A. Ozdaglar, and A. Tahbaz-Salehi. Systemic risk and stability in financial networks. Americal Economic Review, to appear, 2014.

[3] V. V. Acharya and T. Yorulmazer. Information contagion and bank herding. Journal of Money, Credit and Banking, 40(1):215-231, 2008.

[4] D. Aldous. Interacting particle systems as stochastic social dynamics. Bernoulli, 19(4):1122-1149, 2013.

[5] H. Amini. Bootstrap percolation and diffusion in random graphs with given vertex degrees. Electronic Journal of Combinatorics, 17: R25, 2010.

[6] H. Amini. Bootstrap percolation in living neural networks. Journal of Statistcal Physics, 141:459-475, 2010.

[7] H. Amini, R. Cont, and A. Minca. Stress testing the resilience of financial networks. International Journal of Theoretical and Applied Finance, 15(1), 2012.

[8] H. Amini, R. Cont, and A. Minca. Resilience to contagion in financial networks. Mathematical Finance, to appear, 2013.

[9] H. Amini, D. Filipovic, and A. Minca. Systemic risk and central counterparty clearing. Swiss Finance Institute Research Paper, (13-34), 2013.

[10] H. Amini and N. Fountoulakis. Bootstrap percolation in power-law random graphs. Journal of Statistcal Physics, 155(1):72-92, 2014.

[11] H. Amini, N. Fountoulakis, and K. Panagiotou. Bootstrap percolation in inhomogeneous random graphs. Preprint available at http://arxiv.org/abs/1402.2815, 2014.

[12] H. Amini and A. Minca. Mathematical modeling of systemic risk. In E. Kranakis, editor, Advances in Network Analysis and its Applications, volume 18 of Mathematics in Industry, pages 3-26. Springer Berlin Heidelberg, 2013.

[13] K. Anand, B. Craig, and G. Von Peter. Filling in the blanks: Network structure and interbank contagion. Technical report, BIS, 2014.

[14] K. Anand, P. Gai, and M. Marsili. Rollover risk, network structure and systemic financial crises. Journal of Economic Dynamics and Control, 36(8):1088-1100, 2012. 
[15] K. B. Athreya and P. E. Ney. Branching Processes. Springer-Verlag, 1st edition, 1972.

[16] A. Birch, Z. Liu, and T. Aste. A counterparty risk study of the UK banking system. Working paper, 2014.

[17] M. Bloznelis, F. Gtze, and J. Jaworski. Birth of a strongly connected giant in an inhomogeneous random digraph. Journal of Applied Probability, 49(3):601-611, 09 2012.

[18] L. Blume, D. Easley, J. Kleinberg, R. Kleinberg, and E. Tardos. Network formation in the presence of contagious risk. In Proceedings of the 12th ACM conference on Electronic commerce, pages 1-10. ACM, 2011.

[19] L. Blume, D. Easley, J. Kleinberg, R. Kleinberg, and É. Tardos. Which networks are least susceptible to cascading failures? In Foundations of Computer Science (FOCS), 2011 IEEE 52nd Annual Symposium on, pages 393-402. IEEE, 2011.

[20] B. Bollobás. Random Graphs. Cambridge studies in advanced mathematics. Cambridge University Press, 2nd edition, 2001.

[21] B. Bollobás, S. Janson, and O. Riordan. The phase transition in inhomogeneous random graphs. Random Structures \& Algorithms, 31(1):3-122, 2007.

[22] M. Boss, H. Elsinger, M. Summer, and S. Thurner. Network topology of the interbank market. Quantitative Finance, 4(6):677-684, 2004.

[23] A. Braverman and A. Minca. Networks of common asset holdings: Aggregation and measures of vulnerability. Available at SSRN 2379669, 2014.

[24] T. Britton, M. Deijfen, and F. Liljeros. A weighted configuration model and inhomogeneous epidemics. Journal of Statistical Physics, 145(5):1368-1384, 2011.

[25] F. Caccioli, M. Shrestha, C. Moore, and J. Doyne Farmer. Stability analysis of financial contagion due to overlapping portfolios. Journal of Banking \& Finance, 2014.

[26] F. R. Chung and L. Lu. Complex graphs and networks, volume 107. American mathematical society Providence, 2006.

[27] R. Cont and A. Minca. Credit default swaps and systemic risk. Available at SSRN $2487947,2014$.

[28] R. Cont, A. Moussa, and E. B. Santos. Network structure and systemic risk in banking systems. In J.-P. Fouque and J. Langsam, editors, Handbook of systemic risk. Cambridge University Press, 2012.

[29] R. Cont and L. Wagalath. Running for the exit: distressed selling and endogenous correlation in financial markets. Mathematical Finance, 23(4):718-741, 2013.

[30] B. Craig and G. Von Peter. Interbank tiering and money center banks. Journal of Financial Intermediation, 2014.

[31] M. Draief, A. J. Ganesh, and L. Massoulié. Thresholds for virus spread on networks. Annals of Applied Probability, 18(2):359-378, 2008.

[32] M. Draief and L. Massoulié. Epidemics and rumours in complex networks. Cambridge University Press, New York, NY, USA, 1st edition, 2010.

[33] D. Duffie and H. Zhu. Does a central clearing counterparty reduce counterparty risk? Review of Asset Pricing Studies, 1(1):74-95, 2011.

[34] R. Durrett. Random graph dynamics. Cambridge Series in Statistical and Probabilistic Mathematics. Cambridge University Press, Cambridge, 2007. 
[35] L. Eisenberg and T. H. Noe. Systemic Risk in Financial Systems. Management Science, 47(2):236-249, 2001.

[36] P. Gai and S. Kapadia. Contagion in Financial Networks. Proceedings of the Royal Society A, 466(2120):2401-2423, 2010.

[37] P. Glasserman and H. P. Young. How likely is contagion in financial networks? Journal of Banking and Finance, to appear, 2013.

[38] T. R. Hurd, D. Cellai, H. Cheng, S. Melnik, and Q. Shao. Illiquidity and insolvency: a double cascade model of financial crises. Available at SSRN 2424877, 2014.

[39] S. Janson. On percolation in random graphs with given vertex degrees. Electronic Journal of Probability, 14:86-118, 2009.

[40] S. Janson, T. Łuczak, T. Turova, T. Vallier, et al. Bootstrap percolation on the random graph $g_{-}\{n, p\}$. The Annals of Applied Probability, 22(5):1989-2047, 2012.

[41] M. Lelarge. Diffusion and cascading behavior in random networks. Games and Economic Behavior, 75(2):752-775, 2012.

[42] S. Morris and H. S. Shin. Illiquidity component of credit risk. Working paper, 2009.

[43] J. Muller. Interbank credit lines as a channel of contagion. Journal of Financial Services Research, 29(1):37-60, 2006.

[44] M. E. J. Newman, S. H. Strogatz, and D. J. Watts. Random graphs with arbitrary degree distributions and their applications. Physical Review E, 64:026118, 2001.

[45] L. Rogers and L. A. Veraart. Failure and rescue in an interbank network. Management Science, 59(4):882-898, 2013.

[46] N. Schwartz, R. Cohen, D. ben Avraham, A.-L. Barabási, and S. Havlin. Percolation in directed scale-free networks. Physical Review E, 66(1):015104, Jul 2002.

[47] B. Söderberg. General formalism for inhomogeneous random graphs. Physical Review E, 66:066121, 2002.

[48] R. van der Hofstad. Random Graphs and Complex Networks. 2014. Book in preparation, http://www.win.tue.nl/ rhofstad/NotesRGCN.pdf.

[49] D. J. Watts. A simple model of global cascades on random networks. Proceedings of the National Academy of Sciences of the USA, 99(9):5766-5771, 2002.

[50] J. Yellen. Interconnectedness and systemic risk: Lessons from the financial crisis and policy implications. Board of Governors of the Federal Reserve System at the AEA/AFA Joint Luncheon San Diego, California, 2013.

\section{A Proofs}

This appendix contains the proofs of all lemmas, propositions and theorems in the main text.

\section{Proof of Lemma 3}

Consider the following iterative specification of the cascade. We begin by defining the set function $\Phi$ on $\mathcal{P}([n])$,

$$
\Phi(A):=\left\{i \in[n] \mid C_{i}<\sum_{j \in A}\left(1-R_{j i}\right) L_{j i}\right\}, \forall A \in \mathcal{P}([n]) .
$$


We have

$$
A \subseteq B \Longrightarrow \Phi(A) \subseteq \Phi(B),
$$

and the fixed point equation (3) writes

$$
\mathcal{D}^{*}=\Phi\left(\mathcal{D}^{*}\right) .
$$

We now let the increasing sequence of default sets: $\underline{\mathcal{D}}_{0}:=\mathcal{D}_{0}$, and for $k=1, \ldots, n$, $\underline{\mathcal{D}}_{k}:=\Phi\left(\underline{\mathcal{D}}_{k-1}\right)$. We have that $\underline{\mathcal{D}}_{k-1} \subseteq \underline{\mathcal{D}}_{k}$. Let $k^{*}:=\min \left\{k=1, \ldots, n \mid \underline{\mathcal{D}}_{k-1}=\underline{\mathcal{D}}_{k}\right\}$. Since for $k<k^{*},\left|\underline{\mathcal{D}}_{k} \backslash \underline{\mathcal{D}}_{k-1}\right| \geq 1$, it follows that $k^{*} \leq n$. Moreover, $\underline{\mathcal{D}}^{*}:=\underline{\mathcal{D}}_{k^{*}}$ satisfies the fixed point equation (3). In particular, since $k^{*} \leq n$, it is guaranteed that $\underline{\mathcal{D}}_{k^{*}}=\underline{\mathcal{D}}_{n-1}$.

Similarly, we let the decreasing sequence of default sets: $\overline{\mathcal{D}}_{0}:=[n]$, and for $k=1, \ldots, n$, $\overline{\mathcal{D}}_{k}:=\Phi\left(\overline{\mathcal{D}}_{k-1}\right)$. We have that $\underline{\mathcal{D}}_{k} \subseteq \underline{\mathcal{D}}_{k-1}$. Let $l^{*}:=\min \left\{k=1, \ldots, n \mid \underline{\mathcal{D}}_{k-1}=\underline{\mathcal{D}}_{k}\right\}$. Since for $k<l^{*},\left|\underline{\mathcal{D}}_{k-1} \backslash \underline{\mathcal{D}}_{k}\right| \geq 1$, it follows that $l^{*} \leq n$. Moreover, $\overline{\mathcal{D}}^{*}:=\overline{\mathcal{D}}_{l^{*}}$ satisfies the fixed point equation (3) and $\overline{\mathcal{D}}_{l^{*}}=\overline{\mathcal{D}}_{n-1}$.

It now remains to show that any fixed point of (3) is such that $\underline{\mathcal{D}}^{*} \subseteq \mathcal{D}^{*} \subseteq \overline{\mathcal{D}}^{*}$. We clearly have that

$$
\underline{\mathcal{D}}_{0}=\mathcal{D}_{0} \subseteq \mathcal{D}^{*} \subseteq[n]=\overline{\mathcal{D}}_{0}
$$

Using the monotonicity property (22), we obtain by forward induction that

$$
\underline{\mathcal{D}}_{k} \subseteq \mathcal{D}^{*} \subseteq \overline{\mathcal{D}}_{k}, \forall k=1, \ldots, n-1,
$$

which concludes the proof.

\section{Proof of Proposition 5}

Let $p_{i}=\mathbb{P}\left(i \in \mathcal{C}_{1}\right)$. Hence, $p_{i}=1$ if $i \in \mathcal{D}_{0}$ and otherwise $p_{i} \leq \sum_{j} \beta_{j i} p_{j}$, which writes for all $i=1,2, \ldots, n$ as

$$
p_{i} \leq \mathbb{1}\left(i \in \mathcal{D}_{0}\right)+\sum_{j} \beta_{j i} p_{j}
$$

We thus obtain

$$
\begin{aligned}
\sum_{i} p_{i} & \leq \sum_{i} \mathbb{1}\left(i \in \mathcal{D}_{0}\right)+\sum_{i} \sum_{j} \beta_{j i} p_{j} \\
& =\left|\mathcal{D}_{0}\right|+\sum_{j}\left(\sum_{i} \beta_{j i}\right) p_{j} \\
& \leq\left|\mathcal{D}_{0}\right|+\beta_{\max }^{+} \sum_{j} p_{j} .
\end{aligned}
$$

We conclude $\mathbb{E}\left[\left|\mathcal{C}_{1}\right|\right]=\sum_{i} p_{i} \leq \frac{1}{1-\beta_{\max }^{+}}\left|\mathcal{D}_{0}\right|$. The second statement follows using the Markov inequality. Namely, for any $\omega(n) \rightarrow \infty$ as $n \rightarrow \infty$ we have $\mathbb{P}\left(\left|\mathcal{C}_{1}\right|>\omega(n)\left|\mathcal{D}_{0}\right|\right) \rightarrow 0$ by the Markov inequality.

\section{Proof of Proposition 7}

Recall that from (23) we have

$$
p_{i} \leq \mathbb{1}\left(i \in \mathcal{D}_{0}\right)+\sum_{j} \beta_{j i} p_{j} .
$$


Let $\mathbf{p}=\left[p_{1}, p_{2}, \ldots, p_{n}\right]$ denote the vector with components $p_{i}, \mathbf{1}$ be the vector with all components equal to 1 and $\mathbf{1}_{\mathcal{D}_{0}}$ be the vector with component 1 for $i \in \mathcal{D}_{0}$ and 0 for $i \notin \mathcal{D}_{0}$. By Equation (23), we have

$$
\mathbf{p} \leq \mathbf{1}_{\mathcal{D}_{0}}+B \mathbf{p}
$$

Denoting by $\|\cdot\|$ the Euclidean norm, we have

$$
\|\mathbf{p}\| \leq\left\|\mathbf{1}_{\mathcal{D}_{0}}+B \mathbf{p}\right\| \leq\left\|\mathbf{1}_{\mathcal{D}_{0}}\right\|+\|B \mathbf{p}\| \leq \sqrt{\left|\mathcal{D}_{0}\right|}+\lambda_{\max }(B)\|\mathbf{p}\| .
$$

We thus have for $\lambda_{\max }(B)<1$ that $\|\mathbf{p}\| \leq \frac{\sqrt{\left|\mathcal{D}_{0}\right|}}{1-\lambda_{\max }(B)}$. Furthermore by the Cauchy-Schwarz inequality,

$$
\mathbb{E}\left[\left|\mathcal{C}_{1}\right|\right]=\sum_{i \in[n]} p_{i}=\left\|\mathbf{1}^{T} \mathbf{p}\right\| \leq\left\|\mathbf{1}^{T}\right\|\|\mathbf{p}\|=\sqrt{n}\|\mathbf{p}\| .
$$

We conclude (if $\lambda_{\max }(B)<1$ )

$$
\mathbb{E}\left[\left|\mathcal{C}_{1}\right|\right] \leq \frac{1}{1-\lambda_{\max }(B)} \sqrt{n\left|\mathcal{D}_{0}\right|},
$$

and the second statement follows using the Markov inequality.

\section{Proof of Proposition 9}

Starting the contagion from $\mathcal{D}_{0}$, the expectation of the number of nodes that defaults at first step is at most $\mid \eta(k) \max \left(k,\left|\mathcal{D}_{0}\right|\right)$, while in the second step is at most $\left|\eta(k)^{2} \max \left(k,\left|\mathcal{D}_{0}\right|\right)\right|$ and so on. We thus have

$$
\mathbb{E}\left[\left|\mathcal{C}_{1}\right|\right] \leq\left|\mathcal{D}_{0}\right|+\eta(k) \max \left(k,\left|\mathcal{D}_{0}\right|\right)+\eta(k)^{2} \max \left(k,\left|\mathcal{D}_{0}\right|\right)+\ldots,
$$

and so when $\eta(k)<1$, we obtain $\mathbb{E}\left[\left|\mathcal{C}_{1}\right|\right] \leq \frac{1}{1-\eta(k)} \max \left(k,\left|\mathcal{D}_{0}\right|\right)$. Again, the second statement follows using Markov inequality.

\section{Proofs of Theorems 11, 15}

It is discussed in [17] how to adapt the arguments in [21] to the case of directed inhomogeneous random graphs for the supercritical case when $\left\|T_{\hat{\beta}}^{+}\right\|>1$ (note that $\left\|T_{\hat{\beta}}^{+}\right\|=\lambda_{\max }(\bar{B})$ in the finite-type case). We now give a proof for the subcritical case, i.e., $\left\|T_{\hat{\beta}}^{+}\right\|<1$, by adapting the arguments of [21] to the case of directed inhomogeneous random graphs.

We first consider the case when the set of types is finite. The result will follow by comparing the neighborhood of a vertex in the graph to a subcritical branching process; see Remark 12. Recall that we have $n_{i}$ banks of type $s_{i}$ and $n_{i} / n \rightarrow \mu_{i}$ as $n \rightarrow \infty$. Let $\mathcal{C}(i)$ denote the set of banks that can be reached from bank $i$ through contagious links. Then $j \in \mathcal{C}(i)$ if and only if there is a directed path from $i$ to $j$ by contagious links. We say that bank $i$ is systemically important if and only if the component $\mathcal{C}(i)$ has at least $K \log n$ banks, where $K$ is a large constant to be chosen below. We let $\mathcal{A}$ be the set of systemically important banks, i.e.,

$$
\mathcal{A}:=\{i \in[n]|| \mathcal{C}(i) \mid \geq K \log n\}
$$

Starting from $v \in \mathcal{D}_{0}$ with type $s_{i}$ and for any $\epsilon>0$, we can upper bound the neighborhood of $v$ by the Galton-Watson process $\mathcal{X}_{i}^{+}(1+\epsilon)$ which starts at a particle of type 
$s_{i}$ and such that the number of children of type $s_{k} \in \mathcal{S}$ of a particle of type $s_{j} \in \mathcal{S}$ has a Poisson distribution with mean $(1+\epsilon) \bar{b}_{j k}=(1+\epsilon) \mu_{k} \hat{\beta}\left(s_{j}, s_{k}\right)$, for all $1 \leq j, k \leq r$.

For $\epsilon$ small enough the branching process $\mathcal{X}_{i}^{+}(1+\epsilon)$ is subcritical. Consider the generating function of $\left|(1+\epsilon) \mathcal{X}_{i}^{+}\right|$which is given for any $z>0$ by $G_{i}(z)=\mathbb{E}\left[z^{\left|\mathcal{X}_{i}^{+}(1+\epsilon)\right|}\right]$. Using the independence of the Poisson numbers of particles of each type in the first generation and the fact that the generating function of Poisson distribution with mean $\lambda$ is $\exp (\lambda(z-1))$ we have

$$
G(z)=z \exp (\bar{B}(G(z)-\mathbf{1}))
$$

where $G(z)=\left[G_{1}(z), \ldots, G_{r}(z)\right]^{\prime}$. In the subcritical case when $\lambda_{\max \bar{B}}<1$, it is known (by the implicit function theorem) that this generating function has a finite solution for $z$ in a neighborhood of 1 and it follows that $G_{i}(z)<\infty$ for all $i=1,2, \ldots, r$ when $z<1+\epsilon$. Moreover, there exists $\delta>0$ such that

$$
\mathbb{P}\left(\left|\mathcal{X}_{i}^{+}(1+\epsilon)\right| \geq k\right) \leq e^{-\delta k} .
$$

Choosing $K=2 / \delta$, we conclude that $\mathbb{P}(v \in \mathcal{A}) \leq n^{-2}$ for all $v \in[n]$. By union bound over all banks, we have w.h.p. $|\mathcal{A}|=0$ and thus $\left|\mathcal{C}_{1}\right| /\left|\mathcal{D}_{0}\right|=O_{p}(\log n)$.

For the general case, it suffices to bound $\hat{\beta}=\hat{\beta}^{(n)}$ for large $n$ from the above by a subcritical finite-type kernel $\hat{\beta}^{\prime}$ and the result follows similarly as above; see [21].

\section{Proof of Corollary 21}

Let $\pi_{\epsilon}^{*}$ be the smallest fixed point of $I$ in $[0,1]$, when a fraction $\epsilon$ of all nodes represent fundamental defaults, i.e., this is the smallest solution in $[0,1]$ to the fixed point equation

$$
\pi=I_{\epsilon}(\pi):=\epsilon+\sum_{j, k} \sum_{\theta \geq 1} \frac{k \mu(j, k)}{\lambda} p(j, k, \theta) \mathbb{P}(\operatorname{Bin}(j, \pi) \geq \theta),
$$

and let $\hat{\pi}>0$ be the smallest positive solution of

$$
\pi=I_{0}(\pi):=\sum_{j, k} \sum_{\theta \geq 1} \frac{k \mu(j, k)}{\lambda} p(j, k, \theta) \mathbb{P}(\operatorname{Bin}(j, \pi) \geq \theta) .
$$

We first show that such a solution exists in $(0,1)$. Note that $I_{0}(0)=0, I_{0}(1) \leq 1$ and $I_{0}$ is an increasing function of $\pi$. Then in order to prove the existence of such a positive $\hat{\pi}$ it suffices to show that $I^{\prime}(\pi)>1$ for $\pi$ close to zero.

Claim 24. Assume that for some $\Theta \in \mathbb{N}, \gamma \in \mathbb{R}^{+}$and $\beta \in(2,3)$ :

$$
\sum_{k} \sum_{\theta=1}^{\Theta} k \mu(j, k) p(j, k, \theta) \geq \gamma j^{-\beta+1}
$$

for all $j \in \mathbb{N}$. Then there exists $\pi_{0} \in(0,1)$ such that we have $I_{0}^{\prime}(\pi)>1$ for all $\pi \in\left(0, \pi_{0}\right]$.

Proof. We have for $\pi \in(0,1)$ and $\Delta \in \mathbb{N}$

$$
\begin{aligned}
I_{0}^{\prime}(\pi) & =\sum_{j, k} \sum_{\theta \geq 1} \frac{j k \mu(j, k)}{\lambda} p(j, k, \theta) \mathbb{P}(\operatorname{Bin}(j-1, \pi)=\theta-1) \\
& \geq \frac{1}{\lambda} \sum_{j=\Delta+1}^{2 \Delta} \sum_{k} \sum_{\theta=1}^{\Theta} j k \mu(j, k) p(j, k, \theta) \mathbb{P}(\operatorname{Bin}(j-1, \pi)=\theta-1) .
\end{aligned}
$$


We now set $\pi_{0}=\frac{1}{\Delta}$ so that we have for $\pi \leq \pi_{0}$ and $\Delta$ large enough

$$
\begin{aligned}
I_{0}^{\prime}(\pi) & \geq \frac{1}{\lambda} \sum_{j=\Delta+1}^{2 \Delta} \sum_{k} \sum_{\theta=1}^{\Theta} j k \mu(j, k) p(j, k, \theta) e^{-(j-1) \pi} \frac{((j-1) \pi)^{\theta-1}}{(\theta-1) !} \\
& \geq \frac{e^{-2}}{\lambda(\Theta-1) !} \sum_{j=\Delta+1}^{2 \Delta} j \sum_{k} \sum_{\theta=1}^{\Theta} k \mu(j, k) p(j, k, \theta) \\
& \geq \frac{\gamma e^{-2}}{\lambda(\Theta-1) !} \sum_{j=\Delta+1}^{2 \Delta} j^{-\beta+2} \\
& >\frac{\gamma e^{-2}}{\lambda(\Theta-1) !} \Delta^{-\beta+3} .
\end{aligned}
$$

Hence by choosing $\Delta$ large enough, e.g.,

$$
\Delta \geq\left(\frac{\gamma e^{-2}}{\lambda(\Theta-1) !}\right)^{\frac{1}{3-\beta}},
$$

and setting $\pi_{0}=1 / \Delta$ we have $I_{0}^{\prime}(\pi)>1$ for all $\pi \leq \pi_{0}$ and the claim thus follows.

Since $I_{\epsilon}$ is continuous we have $\lim _{\epsilon \rightarrow 0^{+}} \pi_{\epsilon}^{*}=\hat{\pi}$. The corollary now follows by Theorem 18 .

\section{Proof of Theorem 22}

We adapt the arguments in [10] to consider the default cascade in directed power-law random graphs. Assume that $\left(\bar{d}_{\text {in }}(i)\right)_{i \in[n]}$ follows a power law distribution with parameter $\beta \in(2,3)$, i.e., there are $0<\gamma_{1}<\gamma_{2}, x_{0}>0$ and $0<\zeta \leq \frac{1}{\beta-1}$ such that for all $x_{0} \leq x \leq n^{\zeta}$

$$
\gamma_{1} x^{-\beta+1} \leq 1-F_{\text {in }}^{(n)}(x) \leq \gamma_{2} x^{-\beta+1},
$$

and $F_{\text {in }}^{(n)}(x)=0$ for $x<x_{0}$, but $F_{\text {in }}^{(n)}(x)=1$ for $x>n^{\zeta}$.

Note that $\mathcal{D}_{0} \subseteq \mathcal{C}_{1}$ and, since there is not any contagious link from the banks in $\mathcal{C}_{1}$ to the banks outside of $\mathcal{C}_{1}$, the first default outside $\mathcal{C}_{1}$ happens for a bank $i \in[n] / \mathcal{C}_{1}$ if at least two of the debtors of $j$ are inside $\mathcal{C}_{1}$. We will show in the following that if $\sum_{i \in \mathcal{C}_{1}} \bar{d}_{\text {out }}(i) \ll d_{c}(n):=n^{\frac{1-\zeta(3-\beta)}{2}}$ then w.h.p. there are no bank outside $\mathcal{C}_{1}$ that has at least 2 debtors in $\mathcal{C}_{1}$ and therefore the default cascade process does not actually evolve outside $\mathcal{C}_{1}$.

For every bank $j \in[n] / \mathcal{C}_{1}$, we define an indicator random variable $X_{j}$ which is 1 when bank $j$ has at least 2 debtors in $\mathcal{C}_{1}$ and we let $X=\sum_{j \in[n] / \mathcal{C}_{1}} X_{j}$. We show in the following that $\mathbb{E} X=o(1)$, thus implying that w.h.p. $X=0$.

For $j \in[n] / \mathcal{C}_{1}$ let $p_{j}=\mathbb{E} X_{j}=\mathbb{P}\left(X_{j}=1\right)$, so that we have

$$
p_{j}=\mathbb{P}\left(\sum_{i \in \mathcal{C}_{1}} E_{i j} \geq 2\right),
$$

where $E_{i j}$ is the indicator random variable that is equal to 1 precisely when $L_{i j}>0$. Hence, the random variable $E_{i j}$ is Bernoulli distributed with expected value equal to $\frac{\bar{d}_{\text {out }}(i) \bar{d}_{\text {in }}(j)}{W_{n}}$. 
We now use a Chernoff-bound-like technique to bound this probability. We have for all $\theta>0$ and $j \in[n] / \mathcal{C}_{1}$ :

$$
\begin{aligned}
\mathbb{P}\left(\sum_{i \in \mathcal{C}_{1}} E_{i j} \geq 2\right) & =\mathbb{P}\left(\exp \left(\theta \sum_{i \in \mathcal{C}_{1}} E_{i j}\right) \geq e^{2 \theta}\right) \\
& \leq \frac{\mathbb{E}\left[\exp \left(\theta \sum_{i \in \mathcal{C}_{1}} E_{i j}\right)\right]}{e^{2 \theta}}=\frac{\prod_{i \in \mathcal{C}_{1}} \mathbb{E}\left[\exp \left(\theta E_{i j}\right)\right]}{e^{2 \theta}} \\
& =\frac{\prod_{i \in \mathcal{C}_{1}}\left(e^{\theta} \frac{\bar{d}_{\text {out }}(i) \bar{d}_{\text {in }}(j)}{W_{n}}+1-\frac{\bar{d}_{\text {out }}(i) \bar{d}_{\text {in }}(j)}{W_{n}}\right)}{e^{2 \theta}} \\
& \leq \frac{\prod_{i \in \mathcal{C}_{1}} \exp \left(\left(e^{\theta}-1\right) \frac{\bar{d}_{\text {out }}(i) \bar{d}_{\text {in }}(j)}{W_{n}}\right)}{e^{2 \theta}} \\
& =\frac{\exp \left(\left(e^{\theta}-1\right) \frac{\bar{d}_{\text {in }}(j) \sum_{i \in \mathcal{C}_{1}} \bar{d}_{\text {out }}(i)}{W_{n}}\right)}{e^{2 \theta}} \\
& \ll \exp \left(\left(e^{\theta}-1\right) \frac{\bar{d}_{\text {in }}(j) d_{c}(n)}{\lambda n}-2 \theta\right) .
\end{aligned}
$$

The exponent in the last expression is minimized when $\theta$ is such that $e^{\theta}=\frac{2 \lambda n}{d_{\text {in }}(j) d_{c}(n)}$. Hence we obtain

$$
\begin{aligned}
p_{j}=\mathbb{P}\left(\sum_{i \in \mathcal{C}_{1}} E_{i j} \geq 2\right) & \leq \exp \left(e^{\theta} \frac{\bar{d}_{\text {in }}(j) d_{c}(n)}{\lambda n}\right) e^{-2 \theta} \\
& =\left(\frac{e \bar{d}_{\text {in }}(j) d_{c}(n)}{\lambda n}\right)^{2}
\end{aligned}
$$

Thus we have

$$
\mathbb{E} X \leq \sum_{j \in[n]} p_{j} \ll\left(\frac{e d_{c}(n)}{\lambda n}\right)^{2} \sum_{j \in[n]} \bar{d}_{\text {in }}(j)^{2} .
$$

We now need to give an estimate on $\sum_{j \in[n]} \bar{d}_{\text {in }}(j)^{2}$.

Claim 25. For all integers $r \geq 2$ and for $\beta \in(2,3)$ we have

$$
\sum_{j \in[n]} \bar{d}_{\mathrm{in}}(j)^{2}=\Theta\left(n^{1+\zeta(3-\beta)}\right) .
$$

Proof. By definition, since $\bar{d}_{\text {in }}$ is power-law distributed, there exists a positive real $x_{0}$ such that for every $x_{0} \leq s \leq n^{\zeta}$ we have

$$
\gamma_{1} s^{-\beta+1} \leq 1-F_{\text {in }}^{(n)}(s) \leq \gamma_{2} s^{-\beta+1},
$$

whereas for $s<x_{0}$ we have $F_{\text {in }}^{(n)}(s)=0$ and for $s>n^{\zeta}$ we have $F_{\text {in }}^{(n)}(s)=1$. We define the function $h_{n}$ on $[0,1]$ as follows. For $0 \leq x \leq 1-F_{\text {in }}^{(n)}\left(n^{\zeta}\right)$ we set $h_{n}(x)=n^{\zeta}$ and for 
$1-F_{\text {in }}^{(n)}\left(n^{\zeta}\right)<x \leq 1$ we set $h_{n}(x)=\left[1-F_{\text {in }}^{(n)}\right]^{-1}(x)$. Then we have

$$
\begin{aligned}
\sum_{i \in[n]} \bar{d}_{\mathrm{in}}(j)^{2} & =n \int_{0}^{1} h_{n}^{2}(x) d x=n\left(\int_{0}^{1-F_{\mathrm{in}}^{(n)}\left(n^{\zeta}\right)} h_{n}^{2}(x) d x+\int_{1-F_{\mathrm{in}}^{(n)}\left(n^{\zeta}\right)}^{1} h_{n}^{2}(x) d x\right) \\
& =\Theta\left(n^{1+\zeta(3-\beta)}\right)+n \int_{1-F_{\mathrm{in}}^{(n)}(n \zeta)}^{1} h_{n}^{2}(x) d x .
\end{aligned}
$$

It now suffices to show that the integral on the right-hand side satisfies the bounds of the claim. Let us also define for every $x \in(0,1]$ the functions $h_{1, n}(x)=\inf \left\{s: \gamma_{1} s^{-\beta+1} \leq x\right\}$ and $h_{2, n}(x)=\inf \left\{s: \gamma_{2} s^{-\beta+1} \leq x\right\}$. By $(25)$, for any $x \in\left(1-F_{\text {in }}^{(n)}\left(n^{\zeta}\right), 1\right]$

$$
\left\{s: \gamma_{2} s^{-\beta+1} \leq x\right\} \subseteq\left\{s: 1-F_{\text {in }}^{(n)}(s) \leq x\right\} \subseteq\left\{s: \gamma_{1} s^{-\beta+1} \leq x\right\},
$$

which implies that

$$
h_{1, n}(x) \leq h_{n}(x) \leq h_{2, n}(x) .
$$

Note that $h_{1, n}(x)=\left(\gamma_{1} / x\right)^{\frac{1}{\beta-1}}$ and $h_{2, n}(x)=\left(\gamma_{2} / x\right)^{\frac{1}{\beta-1}}$. We conclude

$$
\int_{1-F_{\mathrm{in}}^{(n)}\left(n^{\zeta}\right)}^{1}\left(\frac{\gamma_{1}}{x}\right)^{\frac{2}{\beta-1}} d x \leq \int_{1-F_{\mathrm{in}}^{(n)}\left(n^{\zeta}\right)}^{1} h_{n}^{2}(x) d x \leq \int_{1-F_{\mathrm{in}}^{(n)}\left(n^{\zeta}\right)}^{1}\left(\frac{\gamma_{2}}{x}\right)^{\frac{2}{\beta-1}} d x .
$$

For $\ell \in\{1,2\}$, since $\beta \in(2,3)$, we have

$$
\begin{aligned}
\int_{1-F_{\mathrm{in}}^{(n)}(n \zeta)}^{1}\left(\frac{\gamma_{\ell}}{x}\right)^{\frac{2}{\beta-1}} d x & =\gamma_{\ell}^{\frac{2}{\beta-1}} \int_{1-F_{\mathrm{in}}^{(n)}(n \zeta)}^{1}\left(\frac{1}{x}\right)^{\frac{2}{\beta-1}} d x \\
& =\gamma_{\ell}^{\frac{2}{\beta-1}} \frac{\beta-1}{r-\beta+1}\left[\left(1-F_{\mathrm{in}}^{(n)}\left(n^{\zeta}\right)\right)^{-\frac{2}{\beta-1}+1}-1\right] .
\end{aligned}
$$

Recall that $1-F_{\text {in }}^{(n)}\left(n^{\zeta}\right)=\Theta\left(n^{-\zeta(\beta-1)}\right)$. Thus through (26) we deduce that for $\beta \in(2,3)$

$$
n \int_{1-F_{\mathrm{in}}^{(n)}\left(n^{\zeta}\right)}^{1} h_{n}^{2}(x) d x=\Theta\left(n^{1+\zeta(3-\beta)}\right) .
$$

The claim now follows.

Substituting this bound into the right-hand side of (24), we obtain:

$$
\mathbb{E}[X]=o\left(\frac{n^{1-\zeta(3-\beta)}}{n^{2}} n^{1+\zeta(3-\beta)}\right)=o(1),
$$

as desired. 